\title{
Complex Random Matrices and Rician Channel Capacity*
}

\author{
T. Ratnarajah"tit R. Vaillancourt* M. Alvo*
}

CRM-3174

October 2004

*This work was partially supported by the Natural Sciences and Engineering Research Council of Canada and the Centre de recherches mathématiques of the Université de Montréal.

${ }^{\dagger}$ Department of Mathematics and Statistics, University of Ottawa, 585 King Edward Ave., Ottawa ON K1N 6N5 Canada

${ }_{\ddagger}^{\ddagger}$ T. Ratnarajah is now with ECIT, Queen’s University of Belfast, Queen’s Road, Queen’s Island, Belfast BT3 9DT, Northern Ireland, UK (t.ratnarajah@ieee.org) 



\begin{abstract}
The eigenvalue densities of complex noncentral Wishart matrices are investigated to study an open problem in information theory. Specifically, the largest, smallest and joint eigenvalue densities of complex noncentral Wishart matrices are derived. These densities are expressed in terms of complex zonal polynomials and invariant polynomials. The connection between the complex Wishart matrix theory and information theory is given. This facilitates the evaluation of the most important information-theoretic measure, the so-called ergodic channel capacity. In particular, the capacity of multipleinput, multiple-output (MIMO) Rician distributed channels is investigated. We consider both spatially correlated and uncorrelated MIMO Rician channels and derive the exact and easily computable tight upper bound formulas for ergodic capacities. Numerical results are also given, which show how the channel correlation degrades the capacity of the communication system.
\end{abstract}

Key words. complex random matrix, complex noncentral Wishart matrix, zonal polynomial, invariant polynomial, Rician distributed MIMO channel, ergodic channel capacity

AMS(MOS) subject classification. 94A15, 94A05, 60E05, 62H10

To appear in Problems of Information Transmission.

\title{
Résumé
}

On étudie la densité des valeurs propres de matrices de Wishart noncentrales complexes en vue d'applications à un problème ouvert de la théorie de l'information. On obtient la densité conjointe de la plus grande et de la plus petite de ces valeurs propres au moyen de polynômes zonaux complexes et de polynômes invariants. On établie la relation entre ces matrices et la théorie de l'information pour évaluer la très importante capacité ergodique d'un canal. On étudie la capacité des canaux riciens distribués à entrées et sorties multiples (MIMO). On considère les canaux correlés et non correlés dans l'espace et l'on obtient facilement la borne supérieure exacte des capacités ergodiques. Les résultats numériques montrent que la corrélation du canal réduit la capacité du système de communication. 



\section{Introduction}

Let an $n \times m(n \geq m)$ complex Gaussian (or normal) random matrix A be distributed as A $\sim$ $\mathcal{C} N\left(M, I_{n} \otimes \Sigma\right)$ with mean $\mathcal{E}\{\mathrm{A}\}=M$ and covariance $\operatorname{cov}\{\mathrm{A}\}=I_{n} \otimes \Sigma$. Here we read the symbol " $"$ " as "is distributed as", $\mathcal{C} N$ denotes the complex normal distribution and $\otimes$ denotes the Kronecker product. The matrix $\mathrm{W}=\mathrm{A}^{H} \mathrm{~A}$ is called a complex noncentral Wishart matrix. If $M=0, \mathrm{~W}$ is called a complex central Wishart matrix.

In this paper, we investigate the densities of the eigenvalues of complex noncentral Wishart matrices and their applications to information theory. In contrast to the literature in [34], we consider that the elements of random matrices are complex Gaussian distributed with arbitrary mean and covariance matrices. This will lead us to consider the beautiful but difficult theory of complex zonal polynomials and invariant polynomials, which are symmetric polynomials in the eigenvalues of a complex matrix [24]. These polynomials enable us to represent the derived densities of the eigenvalues of complex noncentral Wishart matrices as infinite series.

The eigenvalue densities of complex central Wishart matrices, which are studied in [13], [21], [25] and references therein, are represented by complex hypergeometric functions of a matrix argument, which can be expressed in terms of complex zonal polynomials. The density of a complex noncentral Wishart matrix can also be represented by complex hypergeometric functions. However, the eigenvalue densities of complex noncentral Wishart matrices cannot be solved in terms of complex zonal polynomials. Here we derive these densities using invariant polynomials, which are proposed by Davis [6], 7]. These invariant polynomials have two matrix arguments, which extend the single matrix argument of zonal polynomials. We also derive the largest and smallest eigenvalue distributions of complex noncentral Wishart matrices. These distributions are used in hypothesis testing, principal component analysis, canonical correlation analysis, multiple discriminant analysis, etc. (see [24] and [25]).

The theory of complex noncentral Wishart matrices is used to evaluate the capacity of multipleinput, multiple-output (MIMO) wireless communication systems. Note that the capacity of a communication channel expresses the maximum rate at which information can be reliably conveyed by the channel [1]. Let us denote the number of inputs (or transmitters) and the number of outputs (or receivers) of the MIMO wireless communication system by $n_{t}$ and $n_{r}$, respectively, and assume that the channel coefficients are distributed as complex Gaussian and correlated at the transmitter end. Then the MIMO channel can be represented by an $n_{r} \times n_{t}$ complex random matrix $\mathrm{H} \sim \mathcal{C} N\left(M, I_{n_{r}} \otimes \Sigma\right)$, where $\Sigma$ represents the channel correlation at the transmitter end. This means the covariance matrix of the rows of $\mathrm{H}$ are denoted by $\Sigma$ (same for all rows). If $\Sigma=I_{n_{t}}$ (or $\left.\sigma^{2} I_{n_{t}}\right)$, then the channel is called an uncorrelated Rician distributed channel, otherwise it is called a spatially correlated Rician distributed channel. The Rician channel model is used when there is a strong direct signal path (line-of-sight) between the transmitter and the receiver. Hence, the complex channel coefficients are modeled by non-zero mean. This is a typical satellite communication environment.

In [26], we assume the MIMO channel matrix $\mathrm{H}$ is distributed as $\mathrm{H} \sim \mathcal{C} N\left(0, I_{n_{r}} \otimes \Sigma\right)$, which leads to a correlated Rayleigh distributed channel. This is a typical fixed or mobile communication environment. Recent studies show that a MIMO uncorrelated Rayleigh distributed channel $(\Sigma=$ $\left.\sigma^{2} I_{n_{t}}\right)$ achieves almost $n$ more bits per hertz for every $3 \mathrm{~dB}$ increase in signal-to-noise ratio (SNR) compared to a single-input single-output (SISO) system, which achieves only one additional bit per hertz for every $3 \mathrm{~dB}$ increase in SNR, where $n=\min \left\{n_{t}, n_{r}\right\}$, see [10] and [34]. In this context, we may mention a MIMO strategy that offers a tremendous potential for increasing the information capacity of single user wireless communication systems, namely, the Bell-Labs Layered Space-Time (BLAST) architecture, see [9], [30] and references therein. But in many practical situation, channel 
correlation exists due to poor scattering conditions, which degrade capacity (see [26] and references therein).

In [3, the authors studied the correlated Rayleigh channel matrix with larger dimension (asymptotic analysis), which is only an approximation to the practical correlated channel matrix with finite dimension. In [32], the authors quantified the effect of channel correlations on the MIMO Rayleigh channel capacity by employing an abstract scattering model. More recently, an exact ergodic capacity expression for an uncorrelated Rayleigh MIMO channel matrix was derived in [31], which is different from the work of Telatar [34]. They also derived a tight upper bound to the ergodic capacity for correlated MIMO Rayleigh channels. In [28] and [29], we consider both transmitter and receiver correlation effect on the capacity of MIMO Rayleigh channels.

In [15] and [19], the authors first studied the distribution of the largest eigenvalue of $\Sigma^{-1} A A^{H}$, which is a special case of the complex noncentral Wishart matrix studied in this paper and does not required the invariance polynomial representation. Then they used this distribution to analyze the performance of MIMO MRC (maximal ratio combining) systems. Specifically, they obtained closed-form expressions for the outage probability of MIMO MRC systems operating over a Rician fading channel. In [17], the same authors extended their earlier work by studying the impact of correlation on the performance of MIMO MRC systems and on the MIMO channel capacity over Rayleigh fading channels. In [16], again the same authors presented the exact result on the moment generating function (MGF) of the MIMO channel capacity for independent but not necessarily identically distributed Rician paths. In [18], they extended the MIMO Rician channel capacity results in the presence of Rayleigh cochannel interference. In [14], the authors studied the capacity of uncorrelated MIMO Rician channel. In [35], a single-input multiple-output Rician channel model is studied. All these studies were limited to independent Rician fading channels and the impact of spatial correlation was not addressed for MIMO Rician fading channels.

It is clear that Rayleigh channels and additive white Gaussian noise (AWGN) channels are special cases of a more general spatially correlated MIMO Rician channel model. Therefore, analyzing this more general model is a valuable contribution to the literature. In this paper, we first derive the eigenvalues densities of complex noncentral Wishart matrices. Then, using these densities we evaluate the capacity of spatially correlated MIMO Rician channel matrices $\mathrm{H} \sim \mathcal{C} N\left(M, I_{n_{r}} \otimes \Sigma\right)$ with $n_{r} \geq n_{t}$. This will be done by deriving closed-form ergodic capacity formulas and evaluating them numerically.

This paper is organized as follows. Section 2 provides the necessary tools for deriving the distribution theory and channel capacity. The complex noncentral Wishart matrix is studied in Section 3. The capacity of MIMO channels is studied in Section 4 and the computational methods are given in Section 5. Finally, concluding remaks are given in Section 6.

\section{Preliminaries}

The eigenvalue density can be calculated using differential forms on manifold. In addition, the expression of the noncentral multivariate density functions requires integration with respect to Haar measures on locally compact group [24]. In this section we address these issues for the complex multivariate distribution. We start by defining the Stiefel manifold. 


\section{The Stiefel manifold}

The set of all matrices $E \in \mathbb{C}^{n \times m}(n \geq m)$ with orthonormal columns is called the Stiefel manifold, denoted by $\mathcal{C} V_{m, n}$. Thus,

$$
\mathcal{C} V_{m, n}=\left\{E \in \mathbb{C}^{n \times m} ; E^{H} E=I_{m}\right\} .
$$

The elements of $E$ can be regarded as the coordinates of a point on a $\left(2 m n-m^{2}\right)$-dimensional surface in 2mn-dimensional Euclidean space. The volume of the Stiefel manifold $\mathcal{C} V_{m, n}$ is given in [27] by

$$
\operatorname{Vol}\left(\mathcal{C} V_{m, n}\right)=\int_{\mathcal{C} V_{m, n}}\left(E^{H} d E\right)=\frac{2^{m} \pi^{m n}}{\mathcal{C} \Gamma_{m}(n)},
$$

where the complex multivariate gamma function is

$$
\mathcal{C} \Gamma_{m}(a)=\pi^{m(m-1) / 2} \prod_{k=1}^{m} \Gamma(a-k+1), \quad \operatorname{Re}(a)>m-1 .
$$

If $m=n$, then we get a special case of Stiefel manifold, the so-called unitary manifold, defined by

$$
\mathcal{C} V_{m, m}=\left\{E \in \mathbb{C}^{m \times m} ; E^{H} E=I_{m}\right\} \equiv U(m),
$$

where $U(m)$ denotes the group of unitary $m \times m$ matrices. The volume of $U(m)$ is given by

$$
\operatorname{Vol}[U(m)]=\int_{U(m)}\left(E^{H} d E\right)=\frac{2^{m} \pi^{m^{2}}}{\mathcal{C} \Gamma_{m}(m)} .
$$

The differential form

$$
(d E) \triangleq \frac{1}{\operatorname{Vol}[U(m)]}\left(E^{H} d E\right)=\frac{\mathcal{C} \Gamma_{m}(m)}{2^{m} \pi^{m^{2}}}\left(E^{H} d E\right)
$$

has the property that

$$
\int_{U(m)}(d E)=1
$$

and it represents the normalized Haar invariant probability measure on $U(m)$.

\section{Complex zonal polynomials}

First, we define the complex multivariate hypergeometric coefficients $[a]_{\kappa}$ which frequently occur in integrals involving complex zonal polynomials. Let $\kappa=\left(k_{1}, \ldots, k_{m}\right)$ be a partition of the integer $k$ with $k_{1} \geq \cdots \geq k_{m} \geq 0$ and $k=k_{1}+\cdots+k_{m}$. Then [20]

$$
[a]_{\kappa}=\prod_{i=1}^{m}(a-i+1)_{k_{i}},
$$

where $(a)_{k}=a(a+1) \cdots(a+k-1)$ and $(a)_{0}=1$. The complex zonal polynomial ${ }^{4}$ of a complex matrix $X \in \mathbb{C}^{m \times m}$ is defined in [13] as

$$
C_{\kappa}(X)=\chi_{[\kappa]}(1) \chi_{[\kappa]}(X),
$$

${ }^{4}$ Note that in the literature the real and the complex zonal polynomials are denoted by $C_{\kappa}(X)$ and $\widetilde{C}_{\kappa}(X)$, respectively. However, we use $C_{\kappa}(X)$ for the complex case because we are not considering the real case in this paper. 
where $\chi_{[\kappa]}(1)$ is the dimension of the representation $[\kappa]$ of the symmetric group on $k$ symbols given by

$$
\chi_{[\kappa]}(1)=k ! \frac{\prod_{i<j}^{m}\left(k_{i}-k_{j}-i+j\right)}{\prod_{i=1}^{m}\left(k_{i}+m-i\right) !},
$$

and $\chi_{[\kappa]}(X)$ is the character of the representation $[\kappa]$ of the linear group given as a symmetric function of the eigenvalues, $\mu_{1}, \ldots, \mu_{m}$, of $X$ by

$$
\chi_{[\kappa]}(X)=\frac{\operatorname{det}\left[\left(\mu_{i}^{k_{j}+m-j}\right)\right]}{\operatorname{det}\left[\left(\mu_{i}^{m-j}\right)\right]} .
$$

The following basic properties are given in [13]:

$$
(\operatorname{tr} X)^{k}=\sum_{\kappa} C_{\kappa}(X)
$$

and

$$
\int_{U(m)} C_{\kappa}\left(A X B X^{H}\right)(d X)=\frac{C_{\kappa}(A) C_{\kappa}(B)}{C_{\kappa}\left(I_{m}\right)},
$$

where $(d X)$ is the normalized invariant measure on the unitary group $U(m)$ and

$$
C_{\kappa}\left(I_{m}\right)=2^{2 k} k !\left(\frac{1}{2} m\right)_{\kappa} \frac{\prod_{i<j}^{r}\left(2 k_{i}-2 k_{j}-i+j\right)}{\prod_{i=1}^{r}\left(2 k_{i}+r-i\right) !}
$$

with

$$
\left(\frac{1}{2} m\right)_{\kappa}=\prod_{i=1}^{r}\left(\frac{1}{2}(m-i+1)\right)_{k_{i}} .
$$

Note that the partition $\kappa$ of $k$ has $r$ nonzero parts.

\section{Complex hypergeometric functions}

The probability distributions of complex random matrices are often derived in terms of complex hypergeometric functions of matrix arguments. The following two definitions of complex hypergeometric functions with single and double matrix argument are due to Constantine [5].

Definition 1 The hypergeometric function of one complex matrix is defined as ${ }^{5}$

$$
{ }_{p} F_{q}\left(a_{1}, \ldots, a_{p} ; b_{1}, \ldots, b_{q} ; X\right)=\sum_{k=0}^{\infty} \sum_{\kappa} \frac{\left[a_{1}\right]_{\kappa} \cdots\left[a_{p}\right]_{\kappa}}{\left[b_{1}\right]_{\kappa} \cdots\left[b_{q}\right]_{\kappa}} \frac{C_{\kappa}(X)}{k !},
$$

where $X \in \mathbb{C}^{m \times m},\left\{a_{i}\right\}_{i=1}^{p}$ and $\left\{b_{i}\right\}_{i=1}^{q}$ are arbitrary complex numbers, and $\sum_{\kappa}$ denotes summation taken over all partitions of $k$ into $m$ parts.

Note that none of the parameters $b_{i}$ is allowed to be zero or an integer or half-integer $\leq m-1$. Otherwise some of the denominator terms are zero [24].

Remark 1 The convergence of (4) is given in [24]:

\footnotetext{
${ }^{5}$ Note that in the literature the real and the complex hypergeometric functions are denoted by ${ }_{p} F_{q}$ and ${ }_{p} \widetilde{F}_{q}$, respectively. However, we use ${ }_{p} F_{q}$ for the complex case because we are not considering the real case in this paper.
} 
(i) If $p \leq q$, then the series converges for all $X$.

(ii) If $p=q+1$, then the series converges for $\sigma(X)<1$, where the spectral radius, $\sigma(X)$, of $X$ is the maximum of the absolute values of the eigenvalues of $X$.

(iii) If $p>q+1$, then the series diverges for all $X \neq 0$, unless it terminates. Note that the series terminates when some of the numerators $\left[a_{j}\right]_{\kappa}$ in the series vanish.

Special cases are

$$
{ }_{0} F_{0}(X)=\operatorname{etr}(X), \quad{ }_{1} F_{0}(a ; X)=\operatorname{det}(I-X)^{-a}, \quad a \in \mathbb{R},
$$

and

$$
{ }_{0} F_{1}\left(n ; Z Z^{H}\right)=\int_{U(n)} \operatorname{etr}(Z E+\overline{Z E})(d E),
$$

where $Z$ is an $m \times n$ complex matrix with $m \leq n$, etr denotes the exponential of the trace, $\operatorname{etr}(\cdot)=\exp (\operatorname{tr}(\cdot))$, and $\overline{Z E}$ denotes the complex conjugate of $Z E$.

Definition 2 The hypergeometric function of two complex matrices is defined by

$$
{ }_{p} F_{q}^{(m)}\left(a_{1}, \ldots, a_{p} ; b_{1}, \ldots, b_{q} ; X, Y\right)=\sum_{k=0}^{\infty} \sum_{\kappa} \frac{\left[a_{1}\right]_{\kappa} \cdots\left[a_{p}\right]_{\kappa}}{\left[b_{1}\right]_{\kappa} \cdots\left[b_{q}\right]_{\kappa}} \frac{C_{\kappa}(X) C_{\kappa}(Y)}{k ! C_{\kappa}\left(I_{m}\right)},
$$

where $X, Y \in \mathbb{C}^{m \times m}$.

The splitting formula is

$$
\int_{U(m)}{ }_{p} F_{q}\left(A E B E^{H}\right)(d E)={ }_{p} F_{q}^{(m)}(A, B)
$$

\section{Invariant polynomials}

In this section, we describe a class of homogeneous polynomials $C_{\phi}^{\kappa, \tau}(X, Y)$ of degrees $k$ and $t$ in the elements of the $m \times m$ symmetric complex matrices $X$ and $Y$, respectively, (see, [6], [7] and [4]). These polynomials are invariant under the simultaneous transformations

$$
X \rightarrow E^{H} X E, \quad Y \rightarrow E^{H} Y E, \quad E \in U(m) .
$$

Moreover, these polynomials satisfy the following relationship

$$
\int_{U(m)} C_{\kappa}\left(A E^{H} X E\right) C_{\tau}\left(B E^{H} Y E\right)(d E)=\sum_{\phi \in \kappa . \tau} \frac{C_{\phi}^{\kappa, \tau}(A, B) C_{\phi}^{\kappa, \tau}(X, Y)}{C_{\phi}(I)},
$$

where $C_{\kappa}, C_{\tau}$ and $C_{\phi}$ are zonal polynomials, indexed by the ordered partitions $\kappa, \tau$ and $\phi$ of the nonnegative integers $k, t$, and $f=k+t$, respectively, into not more than $m$ parts. If we let $G l(m, \mathbb{C})$ denote the general linear group of $m \times m$ nonsingular complex matrices, then $\phi \in \kappa . \tau$ denotes the irreducible representation of $G l(m, \mathbb{C})$ indexed by $2 \phi$ that occurs in the decomposition of the Kronecker product $2 \kappa \otimes 2 \tau$ of the irreducible representations indexed by $2 \kappa$ and $2 \tau$ [6]. Equation (7) can also be written as

$$
\int_{U(m)} \operatorname{etr}\left(A E^{H} X E+B E^{H} Y E\right)(d E)=\sum_{k, t=0}^{\infty} \sum_{\kappa, \tau ; \phi \in \kappa . \tau} \frac{C_{\phi}^{\kappa, \tau}(A, B) C_{\phi}^{\kappa, \tau}(X, Y)}{k ! t ! C_{\phi}(I)} .
$$

The followings are true: 
- $C_{\phi}^{\kappa, \tau}(X, X)=\theta_{\phi}^{\kappa, \tau} C_{\phi}(X)$, where $\theta_{\phi}^{\kappa, \tau}=C_{\phi}^{\kappa, \tau}(I, I) / C_{\phi}(I)$,

- $C_{\phi}^{\kappa, \tau}(X, I)=\left[\theta_{\phi}^{\kappa, \tau} C_{\phi}(I) / C_{\kappa}(I)\right] C_{\kappa}(X)$,

- $C_{\kappa}^{\kappa, 0}(X, Y)=C_{\kappa}(X)$ and $C_{\tau}^{0, \tau}(X, Y)=C_{\tau}(Y)$,

- $C_{\kappa}(X) C_{\tau}(Y)=\sum_{\phi \in \kappa . \tau} \theta_{\phi}^{\kappa, \tau} C_{\phi}^{\kappa, \tau}(X, Y)$.

The incomplete gamma function can be written [6] as

$$
\begin{array}{r}
\int_{0}^{X} \operatorname{etr}(-A Y)(\operatorname{det} Y)^{n-m} C_{\tau}(B Y)(d Y)=\frac{\mathcal{C} \Gamma_{m}(n) \mathcal{C} \Gamma_{m}(m)}{\mathcal{C} \Gamma_{m}(m+n)}(\operatorname{det} X)^{n} \\
\times \sum_{k=0}^{\infty} \sum_{\kappa ; \phi \in \kappa . \tau} \frac{[n]_{\phi} \theta_{\phi}^{\kappa, \tau} C_{\phi}^{\kappa, \tau}(-A X, B X)}{k ![n+m]_{\phi}}
\end{array}
$$

\section{The Complex Noncentral Wishart Matrices}

In this section, the complex noncentral Wishart distribution is studied. In Subsection 3.1, we derived the joint eigenvalue density of complex noncentral Wishart matrix. The maximum and minimum eigenvalue distributions are derived in Subsections 3.2 and 3.3, respectively.

The definition of the complex noncentral Wishart distribution is as follows.

Definition 3 Let $\mathrm{W}=\mathrm{A}^{H} \mathrm{~A}$, where the $n \times m(n \geq m)$ matrix $\mathrm{A}$ is distributed as $\mathrm{A} \sim \mathcal{C} N\left(M, I_{n} \otimes\right.$ $\Sigma)$. Then $\mathrm{W}$ is said to have the complex noncentral Wishart distribution with $n$ degrees of freedom, covariance matrix $\Sigma$, and matrix of noncentrality parameters $\Omega=\Sigma^{-1} M^{H} M$. We shall write $\mathrm{W} \sim \mathcal{C} W_{m}(n, \Sigma, \Omega)$

Let $\mathrm{W} \sim \mathcal{C} W_{m}(n, \Sigma, \Omega)$ with $n \geq m$. Then the density of $\mathrm{W}$ is given by

$$
f(W)=\frac{1}{\mathcal{C} \Gamma_{m}(n)(\operatorname{det} \Sigma)^{n}} \operatorname{etr}\left(-\Sigma^{-1} W\right)(\operatorname{det} W)^{n-m} \operatorname{etr}(-\Omega)_{0} F_{1}\left(n ; \Omega \Sigma^{-1} W\right),
$$

where $\Omega=\Sigma^{-1} M^{H} M$.

If $M=0$ in Definition 3 and (10) we obtain the distribution of the complex central Wishart matrix, which is denoted by $\mathrm{W} \sim \mathcal{C} W_{m}(n, \Sigma)$ and its density is given by

$$
f(W)=\frac{1}{\mathcal{C} \Gamma_{m}(n)(\operatorname{det} \Sigma)^{n}} \operatorname{etr}\left(-\Sigma^{-1} W\right)(\operatorname{det} W)^{n-m} .
$$

Theorem 1 The complex noncentral Wishart density can be expressed in terms of the complex central Wishart density, i.e.,

$$
\mathcal{C} W_{m}(n, \Sigma, \Omega)=\mathcal{C} W_{m}(n, \Sigma, 0) \sum_{k, t=0}^{\infty} \sum_{\kappa, \tau ; \phi \in \kappa . \tau} \frac{\theta_{\phi}^{\kappa, \tau} C_{\phi}^{\kappa, \tau}\left(-\Omega, \Omega \Sigma^{-1} W\right)}{k ! t ![n]_{\tau}}
$$

where $C_{\phi}^{\kappa, \tau}$ is an invariant polynomial, indexed by the ordered partitions $\kappa, \tau$ and $\phi$ of the nonnegative integers $k, t$, and $f=k+t$, respectively, into not more than $m$ parts and $\theta_{\phi}^{\kappa, \tau}=C_{\phi}^{\kappa, \tau}(I, I) / C_{\phi}(I)$. 
Proof. We have

$$
\begin{aligned}
\mathcal{C} W_{m}(n, \Sigma, \Omega) & =\mathcal{C} W_{m}(n, \Sigma, 0) \operatorname{etr}(-\Omega)_{0} F_{1}\left(n ; \Omega \Sigma^{-1} W\right) \\
& =\mathcal{C} W_{m}(n, \Sigma, 0) \sum_{k, t=0}^{\infty} \sum_{\kappa, \tau} \frac{C_{\kappa}(-\Omega)}{k !} \frac{C_{\tau}\left(\Omega \Sigma^{-1} W\right)}{t ![n]_{\tau}} \\
& =\mathcal{C} W_{m}(n, \Sigma, 0) \sum_{k, t=0}^{\infty} \sum_{\kappa, \tau ; \phi \in \kappa . \tau} \frac{\theta_{\phi}^{\kappa, \tau} C_{\phi}^{\kappa, \tau}\left(-\Omega, \Omega \Sigma^{-1} W\right)}{k ! t ![n]_{\tau}}
\end{aligned}
$$

The proof is complete.

\subsection{Joint eigenvalue density}

Here we consider the joint eigenvalue density of a complex noncentral Wishart matrix. The following proposition is required in the sequel.

Proposition 1 Let $\mathrm{W}$ be an arbitrary $m \times m$ positive definite complex random matrix with density function $f(W)$. Then the joint density function of the eigenvalues, $\lambda_{1}>\cdots>\lambda_{m}>0$, of $\mathrm{W}$ is

$$
f(\Lambda)=\frac{\pi^{m(m-1)}}{\mathcal{C} \Gamma_{m}(m)} \prod_{k<l}^{m}\left(\lambda_{k}-\lambda_{l}\right)^{2} \int_{U(m)} f\left(E \Lambda E^{H}\right)(d E),
$$

where $\Lambda=\operatorname{diag}\left(\lambda_{1}, \ldots, \lambda_{m}\right)$ and $W=E \Lambda E^{H}$ is the eigendecomposition $W$.

The joint eigenvalue density of a complex noncentral Wishart matrix is given by the following theorem.

Theorem 2 Suppose that $n \geq m$ and consider the $m \times m$ positive definite Hermitian matrix $\mathrm{W} \sim$ $\mathcal{C} W_{m}(n, \Sigma, \Omega)$. Then the joint density of the eigenvalues, $\lambda_{1}>\cdots>\lambda_{m}>0$, of $\mathrm{W}$ is

$$
\begin{aligned}
f(\Lambda)= & \frac{\pi^{m(m-1)}(\operatorname{det} \Sigma)^{-n}}{\mathcal{C} \Gamma_{m}(m) \mathcal{C} \Gamma_{m}(n)} \operatorname{etr}(-\Omega) \prod_{k=1}^{m} \lambda_{k}^{n-m} \prod_{k<l}^{m}\left(\lambda_{k}-\lambda_{l}\right)^{2} \\
& \times \sum_{k, t=0}^{\infty} \sum_{\kappa, \tau ; \phi \in \kappa . \tau} \frac{C_{\phi}^{\kappa, \tau}\left(-\Sigma^{-1}, \Omega \Sigma^{-1}\right) C_{\phi}^{\kappa, \tau}(\Lambda, \Lambda)}{k ! t ![n]_{\tau} C_{\phi}\left(I_{m}\right)}
\end{aligned}
$$

where $\Lambda=\operatorname{diag}\left(\lambda_{1}, \ldots, \lambda_{m}\right)$ and $C_{\phi}^{\kappa, \tau}$ is an invariant polynomial, indexed by the ordered partitions $\kappa, \tau$ and $\phi$ of the nonnegative integers $k, t$, and $f=k+t$, respectively, into not more than $m$ parts.

Proof. From Proposition 1 we obtain

$$
\begin{aligned}
f(\Lambda)= & \frac{\pi^{m(m-1)}}{\mathcal{C} \Gamma_{m}(m)} \prod_{k<l}^{m}\left(\lambda_{k}-\lambda_{l}\right)^{2} \int_{U(m)} f\left(E \Lambda E^{H}\right)(d E) \\
= & \frac{\pi^{m(m-1)}(\operatorname{det} \Sigma)^{-n}}{\mathcal{C} \Gamma_{m}(m) \mathcal{C} \Gamma_{m}(n)} \operatorname{etr}(-\Omega) \prod_{k=1}^{m} \lambda_{k}^{n-m} \prod_{k<l}^{m}\left(\lambda_{k}-\lambda_{l}\right)^{2} \\
& \times \int_{U(m)} \operatorname{etr}\left(-\Sigma^{-1} E \Lambda E^{H}\right){ }_{0} F_{1}\left(n ; \Omega \Sigma^{-1} E \Lambda E^{H}\right)(d E) \\
= & \frac{\pi^{m(m-1)}(\operatorname{det} \Sigma)^{-n}}{\mathcal{C} \Gamma_{m}(m) \mathcal{C} \Gamma_{m}(n)} \operatorname{etr}(-\Omega) \prod_{k=1}^{m} \lambda_{k}^{n-m} \prod_{k<l}^{m}\left(\lambda_{k}-\lambda_{l}\right)^{2} \\
& \times \sum_{k, t=0}^{\infty} \sum_{\kappa, \tau} \frac{1}{k ! t ![n]_{\tau}} \int_{U(m)} C_{\kappa}\left(-\Sigma^{-1} E \Lambda E^{H}\right) C_{\tau}\left(\Omega \Sigma^{-1} E \Lambda E^{H}\right)(d E) .
\end{aligned}
$$


The result follows from equation (7).

\subsection{Distribution of $\lambda_{\max }$}

In this subsection, we derive the distribution of the largest eigenvalue, $\lambda_{\max }$, of a complex noncentral Wishart matrix and apply it to hypothesis testing. The following theorem is needed.

Theorem 3 Let $\mathrm{W} \sim \mathcal{C} W_{m}(n, \Sigma, \Omega)(n \geq m)$ and $\Delta$ be an $m \times m$ positive definite matrix. Then the probability $P(W<\Delta)$ is given by

$$
P(W<\Delta)=\frac{\mathcal{C} \Gamma_{m}(m)(\operatorname{det} \Delta)^{n} \operatorname{etr}(-\Omega)}{\mathcal{C} \Gamma_{m}(n+m)(\operatorname{det} \Sigma)^{n}} \sum_{k, t=0}^{\infty} \sum_{\kappa, \tau ; \phi \in \kappa . \tau} \frac{[n]_{\phi} \theta_{\phi}^{\kappa, \tau} C_{\phi}^{\kappa, \tau}\left(-\Sigma^{-1} \Delta, \Omega \Sigma^{-1} \Delta\right)}{k ! t ![n]_{\tau}[n+m]_{\phi}}
$$

where $C_{\phi}^{\kappa, \tau}$ is an invariant polynomial, indexed by the ordered partitions $\kappa, \tau$ and $\phi$ of the nonnegative integers $k, t$, and $f=k+t$, respectively, into not more than $m$ parts and $\theta_{\phi}^{\kappa, \tau}=C_{\phi}^{\kappa, \tau}(I, I) / C_{\phi}(I)$.

Proof. Using the complex noncentral Wishart density (10) we can write $P(W<\Delta)$ as

$$
\begin{aligned}
P(W<\Delta) & =\frac{\operatorname{etr}(-\Omega)}{\mathcal{C} \Gamma_{m}(n)(\operatorname{det} \Sigma)^{n}} \int_{0}^{\Delta} \operatorname{etr}\left(-\Sigma^{-1} W\right)(\operatorname{det} W)^{n-m}{ }_{0} F_{1}\left(n ; \Omega \Sigma^{-1} W\right)(d W) \\
& =\frac{\operatorname{etr}(-\Omega)}{\mathcal{C} \Gamma_{m}(n)(\operatorname{det} \Sigma)^{n}} \sum_{t=0}^{\infty} \sum_{\tau} \int_{0}^{\Delta} \frac{\operatorname{etr}\left(-\Sigma^{-1} W\right)(\operatorname{det} W)^{n-m} C_{\tau}\left(\Omega \Sigma^{-1} W\right)}{t ![n]_{\tau}}(d W) .
\end{aligned}
$$

The result follows from equation (9).

The following corollary to Theorem 3 describes the distribution of $\lambda_{\max }$.

Corollary 1 If $W \sim \mathcal{C} W_{m}(n, \Sigma, \Omega)$ and $\lambda_{\max }$ is the largest eigenvalue of $W$, then its distribution is given by

$$
P\left(\lambda_{\max }<y\right)=\frac{y^{m n} \mathcal{C} \Gamma_{m}(m) \operatorname{etr}(-\Omega)}{\mathcal{C} \Gamma_{m}(n+m)(\operatorname{det} \Sigma)^{n}} \sum_{k, t=0}^{\infty} \sum_{\kappa, \tau ; \phi \in \kappa . \tau} \frac{[n]_{\phi} \theta_{\phi}^{\kappa, \tau} C_{\phi}^{\kappa, \tau}\left(-y \Sigma^{-1}, y \Omega \Sigma^{-1}\right)}{k ! t ![n]_{\tau}[n+m]_{\phi}},
$$

where $C_{\phi}^{\kappa, \tau}$ is an invariant polynomial, indexed by the ordered partitions $\kappa, \tau$ and $\phi$ of the nonnegative integers $k, t$, and $f=k+t$, respectively, into not more than $m$ parts, and $\theta_{\phi}^{\kappa, \tau}=C_{\phi}^{\kappa, \tau}(I, I) / C_{\phi}(I)$. The density of $\lambda_{\max }$ is obtained by differentiating (16) with respect to $y$.

Proof. The inequality $\lambda_{\max }<y$ is equivalent to $W<y I$. Therefore, the result follows by letting $\Delta=y I$ in Theorem 3 .

The distributional result in Corollary 1 can be used to test hypotheses about $\Sigma$ using statistics which are functions of $\lambda_{\max }$. For example, consider the null hypothesis $H_{0}: \Sigma=I_{m}$. A test on size $\alpha$ based on the largest eigenvalue $\lambda_{\max }$ is to reject $H_{0}$ if $\lambda_{\max }>\lambda(\alpha, m, n)$, where $\lambda(\alpha, m, n)$ is the upper $100 \alpha \%$ point of the distribution of $\lambda_{\max }$ when $\Sigma=I_{m}$, i.e., $P_{I_{m}}\left(\lambda_{\max }>\lambda(\alpha, m, n)\right)=\alpha$. The power function of this test is given by

$$
\beta(\Sigma)=P_{\Sigma}\left(\lambda_{\max }>\lambda(\alpha, m, n)\right)
$$

which depends on $\Sigma$ only through its eigenvalues. The percentage points and power can be computed using the distribution function given in Corollary 1 . 


\subsection{Distribution of $\lambda_{\min }$}

In this subsection, we derive the distribution of the smallest eigenvalue, $\lambda_{\min }$, of a complex noncentral Wishart matrix. This distribution can also be used to test the structure of the covariance matrix $\Sigma$, as explained in the previous subsection. In addition, the distribution of $\lambda_{\min }$ is useful in the area of the principal component analysis. Here it would be of interest to find out the number of eigenvalues which are significant in $\Sigma$. The following theorem is used to derive the distribution of $\lambda_{\min }$.

Theorem 4 If $W \sim \mathcal{C} W_{m}(n, \Sigma, \Omega)(n \geq m)$ and $\Delta$ is an $m \times m$ positive definite matrix, then the probability $P(W>\Delta)$ can be written as

$$
\begin{aligned}
& P(W>\Delta)=\frac{\operatorname{etr}(-\Omega) \operatorname{etr}\left(-\Sigma^{-1} \Delta\right)(\operatorname{det} \Delta)^{n}}{\mathcal{C} \Gamma_{m}(n)(\operatorname{det} \Sigma)^{n}} \sum_{t=0}^{\infty} \sum_{\tau} \frac{1}{t ![n]_{\tau}} \\
& \quad \times \int_{X>0} \operatorname{etr}\left(-\Delta^{1 / 2} \Sigma^{-1} \Delta^{1 / 2} X\right) \operatorname{det}(I+X)^{n-m} C_{\tau}\left(\Omega \Sigma^{-1} \Delta^{1 / 2}(I+X) \Delta^{1 / 2}\right)(d X),
\end{aligned}
$$

where $\tau$ is a partition of $t$.

Proof. Using the complex noncentral Wishart density (10) we can write $P(W>\Delta)$ as

$$
P(W>\Delta)=\frac{\operatorname{etr}(-\Omega)}{\mathcal{C} \Gamma_{m}(n)(\operatorname{det} \Sigma)^{n}} \int_{W>\Delta} \operatorname{etr}\left(-\Sigma^{-1} W\right)(\operatorname{det} W)^{n-m}{ }_{0} F_{1}\left(n ; \Omega \Sigma^{-1} W\right)(d W) .
$$

The change of variable $W=\Delta^{1 / 2}(I+X) \Delta^{1 / 2}$ leads to the differential form $(d W)=(\operatorname{det} \Delta)^{m}(d X)$. Hence,

$$
\begin{aligned}
& P(W>\Delta)=\frac{\operatorname{etr}(-\Omega) \operatorname{etr}\left(-\Sigma^{-1} \Delta\right)(\operatorname{det} \Delta)^{n}}{\mathcal{C} \Gamma_{m}(n)(\operatorname{det} \Sigma)^{n}} \\
& \quad \times \int_{X>0} \operatorname{etr}\left(-\Delta^{1 / 2} \Sigma^{-1} \Delta^{1 / 2} X\right) \operatorname{det}(I+X)^{n-m}{ }_{0} F_{1}\left(n ; \Omega \Sigma^{-1} \Delta^{1 / 2}(I+X) \Delta^{1 / 2}\right)(d X) .
\end{aligned}
$$

The result follows by expanding ${ }_{0} F_{1}\left(n ; \Omega \Sigma^{-1} \Delta^{1 / 2}(I+X) \Delta^{1 / 2}\right)$ in terms of zonal polynomials.

The distribution of the smallest eigenvalue is given in the following corollary.

Corollary 2 If $\mathrm{W} \sim \mathcal{C} W_{m}(n, \Sigma, \Omega)$ and $\lambda_{\min }$ is the smallest eigenvalue of $\mathrm{W}$, then

$$
\begin{aligned}
& P\left(\lambda_{\min }>y\right)=\frac{y^{m n} \operatorname{etr}(-\Omega) \operatorname{etr}\left(-y \Sigma^{-1}\right)}{\mathcal{C} \Gamma_{m}(n)(\operatorname{det} \Sigma)^{n}} \sum_{t=0}^{\infty} \sum_{\tau} \frac{1}{t ![n]_{\tau}} \\
& \quad \times \int_{X>0} \operatorname{etr}\left(-y \Sigma^{-1} X\right) \operatorname{det}(I+X)^{n-m} C_{\tau}\left(y \Omega \Sigma^{-1}(I+X)\right)(d X),
\end{aligned}
$$

where $\tau$ is a partition of $t$. The density of $\lambda_{\min }$ is obtained by differentiating (18) with respect to $y$ and then changing the sign.

Proof. The inequality $\lambda_{\min }>y$ is equivalent to $W>y I$. Therefore, the result follows by letting $\Delta=y I$ in Theorem 4 . 


\section{The MIMO Channel Capacity}

In recent years, multiple-antenna techniques have become a pervasive idea that promises extremely high spectral efficiency for wireless communications. Two issues of concern here are:

(i) the information-theoretic aspects of data transmission using MIMO techniques with emphasis on spectral efficiency and information capacity,

(ii) the practical feasibility of these techniques, aimed at the realization of a significant portion of the capacity promised by information theory, see [9], [30] and references therein.

The basic information theory result reported in the pioneering papers by Foschini and Gans [10] and Telatar [34] showed that enormous spectral efficiency can be achieved through the use of multipleantenna systems. The major conclusion of their work is that the capacity of a MIMO system far exceeds that of a single-antenna system. In particular, in a Rayleigh flat fading environment, a MIMO link has an asymptotic capacity that increases linearly with the number of transmitter and receiver antennas, provided that the complex-valued propagation coefficients between all pairs of transmitter and receiver antennas are statistically independent and known to the receiver antenna array. Here we consider a more general MIMO Rician fading environment and the results presented in the first part of this paper are used to evaluate the capacity of this wireless communication systems. A MIMO channel can be represented by an $n_{r} \times n_{t}$ complex random matrix $\mathrm{H}$, where $n_{t}$ and $n_{r}$ are the number of inputs (or transmitters) and outputs (or receivers) of the communication system, respectively, as shown in Figure 1.

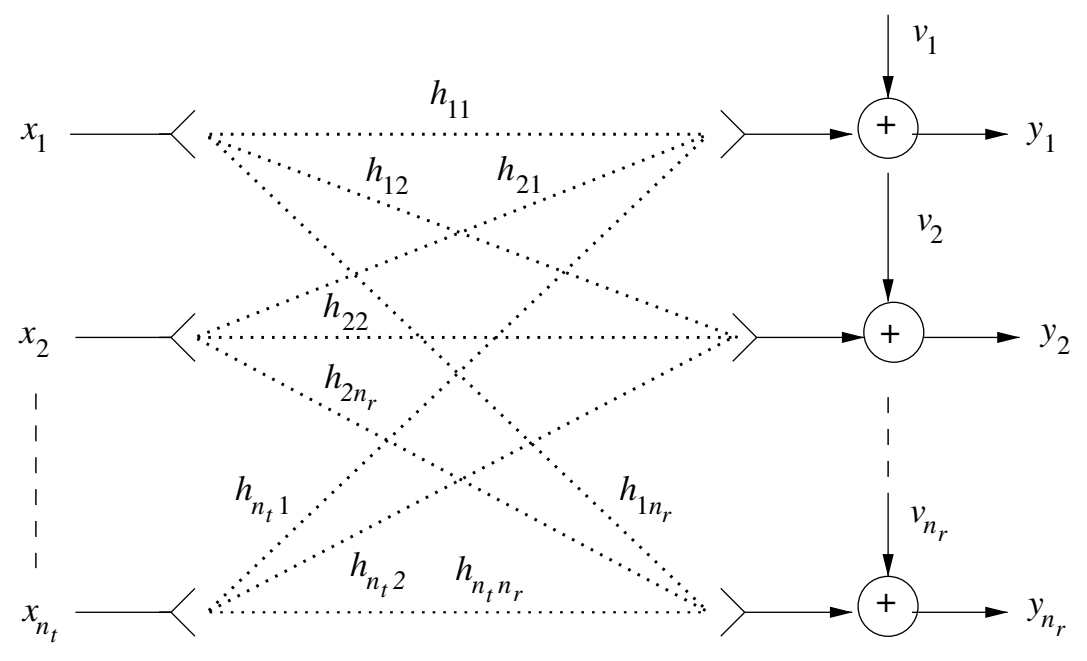

Figure 1: A MIMO communication system.

The complex signal received at the $j$ th output can be written as

$$
y_{j}=\sum_{i=1}^{n_{t}} h_{i j} x_{i}+v_{j},
$$

where $h_{i j}$ is the complex channel coefficient between input $i$ and output $j, x_{i}$ is the complex signal at the $i$ th input and $v_{j}$ is complex Gaussian noise with unit variance. The signal vector received at the output can be written as

$$
\left[\begin{array}{c}
y_{1} \\
\vdots \\
y_{n_{r}}
\end{array}\right]=\left[\begin{array}{ccc}
h_{11} & \cdots & h_{n_{t} 1} \\
\vdots & \vdots & \vdots \\
h_{1 n_{r}} & \cdots & h_{n_{t} n_{r}}
\end{array}\right]\left[\begin{array}{c}
x_{1} \\
\vdots \\
x_{n_{t}}
\end{array}\right]+\left[\begin{array}{c}
v_{1} \\
\vdots \\
v_{n_{r}}
\end{array}\right]
$$


i.e., in vector notation,

$$
y=H x+v,
$$

where $y, v \in \mathbb{C}^{n_{r}}, H \in \mathbb{C}^{n_{r} \times n_{t}}, x \in \mathbb{C}^{n_{t}}$ and $v \sim \mathcal{C} N\left(0, I_{n_{r}}\right)$. It should be noted that the noise $v$ is independent of the input signal $x$ and channel matrix $H$. The total input power is constrained to $\rho$,

$$
\mathcal{E}\left\{x^{H} x\right\} \leq \rho \quad \text { or } \quad \operatorname{tr} \mathcal{E}\left\{x x^{H}\right\} \leq \rho .
$$

We shall deal exclusively with the linear model (20) and compute the capacity of different MIMO channel models in the sequel. We are particularly interested in two-channel models, namely the Rayleigh and Rician distributed channels. The following proposition defines those channel models [23].

Proposition 2 Let $z=r e^{i \theta} \sim \mathcal{C} N\left(\mu_{z}, \sigma^{2}\right)$ denote an element $h_{i j}$ of matrix $H$, where

$$
\mathcal{E}\{z\}=\mu_{z}=\left|\mu_{z}\right| \exp (i \phi), \quad \operatorname{var}\{z\}=\mathcal{E}\left|z-\mu_{z}\right|^{2}=\sigma^{2}
$$

and

$$
f(z)=\frac{1}{\pi \sigma^{2}} \exp \left(\frac{-\left|z-\mu_{z}\right|^{2}}{\sigma^{2}}\right) .
$$

Then the joint density $g\left(r, \theta \mid \mu_{z}, \sigma^{2}\right)$ of $r$ and $\theta$ is given by

$$
g\left(r, \theta \mid \mu_{z}, \sigma^{2}\right)=\frac{r}{\pi \sigma^{2}} \exp \left(\frac{-\left(r^{2}+\left|\mu_{z}\right|^{2}\right)}{\sigma^{2}}\right) \exp \left(\frac{2\left|\mu_{z}\right| r \cos (\theta-\phi)}{\sigma^{2}}\right) .
$$

Therefore, the density of the magnitude or envelope $r$ is given by

$$
\begin{aligned}
h\left(r \mid \mu_{z}, \sigma^{2}\right) & =\frac{r}{\pi \sigma^{2}} \exp \left(\frac{-\left(r^{2}+\left|\mu_{z}\right|^{2}\right)}{\sigma^{2}}\right) \int_{0}^{2 \pi} \exp \left(\frac{2\left|\mu_{z}\right| r \cos (\theta-\phi)}{\sigma^{2}}\right) d \theta \\
& = \begin{cases}\frac{2 r}{\sigma^{2}} \exp \left(\frac{-\left(r^{2}+\left|\mu_{z}\right|^{2}\right)}{\sigma^{2}}\right) I_{0}\left(\frac{2\left|\mu_{z}\right| r}{\sigma^{2}}\right) & r \geq 0 \\
0 & r<0,\end{cases}
\end{aligned}
$$

where $I_{0}$ is the modified Bessel function of the first kind and order zero. The density of the phase $\theta$ is given by

$$
\begin{aligned}
k\left(\theta \mid \mu_{z}, \sigma^{2}\right) & =\frac{1}{\pi \sigma^{2}} \exp \left(\frac{-\left|\mu_{z}\right|^{2}}{\sigma^{2}}\right) \int_{0}^{\infty} r \exp \left(\frac{-r^{2}}{\sigma^{2}}\right) \exp \left(\frac{2\left|\mu_{z}\right| r \cos (\theta-\phi)}{\sigma^{2}}\right) d r \\
& =\left\{\begin{array}{l}
\frac{1}{2 \pi} \exp \left(\frac{-\left|\mu_{z}\right|^{2}\left[1+\sin ^{2}(\theta-\phi)\right]}{2 \sigma^{2}}\right) D_{-2}\left(-\left|\mu_{z}\right|\left(\frac{2}{\sigma^{2}}\right)^{1 / 2} \cos (\theta-\phi)\right) \\
0 \leq \theta<2 \pi \\
0 \text { otherwise, }
\end{array}\right.
\end{aligned}
$$

where $D_{-v}$ is the parabolic cylinder function. If $\mu_{z}=0$, then the density $h\left(r \mid \sigma^{2}\right)$ is given by

$$
h\left(r \mid \sigma^{2}\right)= \begin{cases}\frac{2 r}{\sigma^{2}} \exp \left(\frac{-r^{2}}{\sigma^{2}}\right) & r \geq 0, \\ 0 & r<0 .\end{cases}
$$

In this case, the distribution of the phase $\theta$ is uniform and its density is given by

$$
k\left(\theta \mid \sigma^{2}\right)= \begin{cases}\frac{1}{2 \pi} & 0 \leq \theta<2 \pi \\ 0 & \text { otherwise. }\end{cases}
$$


Definition 4 The density $h\left(r \mid \sigma^{2}\right)$ is called the Rayleigh density and the density $h\left(r \mid \mu_{z}, \sigma^{2}\right)$ is called the Rician density. The Rician $K$-factor is defined as the ratio of the deterministic power to the scattered power, i.e., $K$-factor $=\left|\mu_{z}\right|^{2} / \sigma^{2}$.

We assume that the matrix $\mathrm{H}$ is a complex Gaussian random matrix and the realization of $\mathrm{H}$ is known to the receiver, or equivalently, the channel output consists of the pair $(y, \mathrm{H})$. Note that the transmitter does not know the channel and the input power is distributed equally over all transmitting antennas, which is a natural thing to do in this case. Moreover, if we assume a block-fading model and coding over many independent fading intervals, then the Shannon or ergodic capacity of the random MIMO channel [34] is given by

$$
C=\mathcal{E}_{\mathrm{H}}\left\{\log \operatorname{det}\left(I_{n_{t}}+\left(\rho / n_{t}\right) \mathrm{H}^{H} \mathrm{H}\right)\right\}=\mathcal{E}_{\mathrm{W}}\left\{\log \operatorname{det}\left(I_{n_{t}}+\left(\rho / n_{t}\right) \mathrm{W}\right)\right\} .
$$

According to Proposition 2, if $\mathrm{W} \sim \mathcal{C} W_{n_{t}}\left(n_{r}, \Sigma, \Omega\right)$ then the channel is Rician distributed and if $\mathrm{W} \sim \mathcal{C} W_{n_{t}}\left(n_{r}, \Sigma\right)$ then the channel is Rayleigh distributed. The capacities of Rician and Rayleigh distributed MIMO channels are computed using the complex noncentral and central Wishart densities given in equations (10) and (11), respectively. The computation of correlated and uncorrelated Rayleigh channel capacities are studied in [26] and [34], respectively.

Here we evaluate the channel capacity of a Rician channel. In a Rician channel, the distribution of an $n_{r} \times n_{t}$ channel matrix $\mathrm{H}$ is given by $\mathrm{H} \sim \mathcal{C} N\left(M, I_{n_{r}} \otimes \Sigma\right)$, with $n_{r} \geq n_{t}$. Here the covariance matrix of the rows of $\mathrm{H}$ is denoted by $\Sigma$, which is an $n_{t} \times n_{t}$ Hermitian matrix. Note that the offdiagonal elements of $\Sigma$ are nonzero for correlated channels. In other words, the channel coefficients from different transmitter antennas to a single receiver antenna is correlated (correlated at the transmitter end). The channel capacity is given by the following theorem.

Theorem 5 Consider a Rician channel, i.e., $\mathrm{H} \sim \mathcal{C} N\left(M, I_{n_{r}} \otimes \Sigma\right)$, with $n_{r} \geq n_{t}$. If the input power is constrained by $\rho$, then the capacity is given by

$$
C=K_{1} \int_{W>0} \log \operatorname{det}\left[I_{n_{t}}+\frac{\rho}{n_{t}} W\right](\operatorname{det} W)^{n_{r}-n_{t}} \operatorname{etr}\left(-\Sigma^{-1} W\right){ }_{0} F_{1}\left(n_{r} ; \Omega \Sigma^{-1} W\right)(d W)
$$

where $W=H^{H} H, \Omega=\Sigma^{-1} M^{H} M$ and

$$
K_{1}=\frac{\operatorname{etr}(-\Omega)}{\mathcal{C} \Gamma_{n_{t}}\left(n_{r}\right)(\operatorname{det} \Sigma)^{n_{r}}} .
$$

Proof. The result follows by evaluating the expectation in equation (26) using the complex noncentral Wishart density given in equation (10).

The following theorem expresses the Rician channel capacity in terms of the eigenvalue density of a complex noncentral Wishart matrix.

Theorem 6 Consider a Rician channel, i.e., $\mathrm{H} \sim \mathcal{C} N\left(M, I_{n_{r}} \otimes \Sigma\right)$, with $n_{r} \geq n_{t}$. If the input power is constrained by $\rho$, then using the joint eigenvalue density of the complex noncentral Wishart matrix $\mathrm{W}=\mathrm{H}^{H} \mathrm{H}$ we can write the capacity as

$$
C=\int_{\Lambda>0} \log \left\{\prod_{k=1}^{n_{t}}\left[1+\frac{\rho}{n_{t}} \lambda_{k}\right]\right\} f(\Lambda) d \Lambda,
$$

where $\lambda_{1}>\cdots>\lambda_{n_{t}}>0$ are the eigenvalues of $\mathrm{W}, \Lambda=\operatorname{diag}\left(\lambda_{1}, \ldots, \lambda_{n_{t}}\right)$ and

$$
\begin{aligned}
f(\Lambda)= & \frac{\pi^{n_{t}\left(n_{t}-1\right)}(\operatorname{det} \Sigma)^{-n_{r}}}{\mathcal{C} \Gamma_{n_{t}}\left(n_{t}\right) \mathcal{C} \Gamma_{n_{t}}\left(n_{r}\right)} \operatorname{etr}(-\Omega) \prod_{k=1}^{n_{t}} \lambda_{k}^{n_{r}-n_{t}} \prod_{k<l}^{n_{t}}\left(\lambda_{k}-\lambda_{l}\right)^{2} \\
& \times \sum_{k, t=0}^{\infty} \sum_{\kappa, \tau ; \phi \in \kappa, \tau} \frac{C_{\phi}^{\kappa, \tau}\left(-\Sigma^{-1}, \Omega \Sigma^{-1}\right) C_{\phi}^{\kappa, \tau}(\Lambda, \Lambda)}{k ! t !\left[n_{r}\right]_{\tau} C_{\phi}\left(I_{n_{t}}\right)} .
\end{aligned}
$$


Proof. From equation (26), the capacity $C$ is given by

$$
C=\mathcal{E}_{\mathrm{W}}\left\{\log \operatorname{det}\left(I_{n_{t}}+\frac{\rho}{n_{t}} \mathrm{~W}\right)\right\}=\mathcal{E}_{\Lambda}\left\{\log \left(\prod_{k=1}^{n_{t}}\left[1+\frac{\rho}{n_{t}} \lambda_{k}\right]\right)\right\} .
$$

The result follows by evaluating equation (30) using the eigenvalue density given in equation (14).

The Rician channel capacity formulas given in Theorems 5 and 6 are difficult to compute. This difficulty motivates us to consider the approximate capacity evaluation, or specifically, finding an upper bound on the Rician capacity, which is studied next. The following lemma is required in the sequel.

Lemma 1 Let $X$ be an $n \times m(n \geq m)$ complex matrix of rank $m$. Then the following inequality holds:

$$
{ }_{0} F_{1}\left(n ; X^{H} X\right) \leq{ }_{0} F_{0}\left(X^{H} X / n\right)
$$

with equality as $n \rightarrow \infty$.

Proof. Since $X^{H} X$ is an $m \times m$ Hermitian positive definite matrix, it is enough to show the following inequality:

$$
\left(\operatorname{det} X^{H} X\right)^{n-m} F_{1}\left(n ; X^{H} X\right) \leq\left(\operatorname{det} X^{H} X\right)^{n-m}{ }_{0} F_{0}\left(X^{H} X / n\right) .
$$

The proof is by contradiction. Suppose, to the contrary, that

$$
\left(\operatorname{det} X^{H} X\right)^{n-m}{ }_{0} F_{1}\left(n ; X^{H} X\right)>\left(\operatorname{det} X^{H} X\right)^{n-m}{ }_{0} F_{0}\left(X^{H} X / n\right) \text {. }
$$

The Laplace transform of the left side of $(33)$ is

$$
\begin{aligned}
g_{L}(Z) & =\int_{X^{H} X>0} \operatorname{etr}\left(-X^{H} X Z\right)\left(\operatorname{det} X^{H} X\right)^{n-m}{ }_{0} F_{1}\left(n ; X^{H} X\right)\left(d X^{H} X\right) \\
& =\mathcal{C} \Gamma_{m}(n)(\operatorname{det} Z)^{-n}{ }_{1} F_{1}\left(n ; n ; Z^{-1}\right) \\
& =\mathcal{C} \Gamma_{m}(n)(\operatorname{det} Z)^{-n}{ }_{0} F_{0}\left(Z^{-1}\right) \\
& =\mathcal{C} \Gamma_{m}(n)(\operatorname{det} Z)^{-n} \operatorname{etr}\left(Z^{-1}\right),
\end{aligned}
$$

where the second equality in equation (34) follows from the complex counterpart of the real case given in [24, Theorem 7.3.4]. The Laplace transform of the right side of (33) is

$$
\begin{aligned}
g_{R}(Z) & =\int_{X^{H} X>0} \operatorname{etr}\left(-X^{H} X Z\right)\left(\operatorname{det} X^{H} X\right)^{n-m}{ }_{0} F_{0}\left(X^{H} X / n\right)\left(d X^{H} X\right) \\
& =\mathcal{C} \Gamma_{m}(n)(\operatorname{det} Z)^{-n}{ }_{1} F_{0}\left(n ; Z^{-1} / n\right) \\
& =\mathcal{C} \Gamma_{m}(n)(\operatorname{det} Z)^{-n} \operatorname{det}\left(I-Z^{-1} / n\right)^{-n} .
\end{aligned}
$$

Since the left and right sides of (33) are both positive, then inequality (33) implies that

$$
g_{L}(Z)>g_{R}(Z)
$$

Thus, comparing equations (34) and (35), we have

$$
\operatorname{det}\left(I-Z^{-1} / n\right)^{-n}<\operatorname{etr}\left(Z^{-1}\right),
$$


that is,

$$
\left(\prod_{k=1}^{m}\left[1-\frac{\varphi_{k}}{n}\right]\right)^{-n}<\exp \left(\sum_{k=1}^{m} \varphi_{k}\right),
$$

Here we may assume that the eigenvalues of the matrix $Z^{-1}$ satisfy $1>\varphi_{1}>\cdots>\varphi_{m}>0$ by taking $Z$ satisfying $Z>Z_{0}>0$, with eigenvalues larger than 1, to ensure convergence of the Laplace transforms in (34) and (35). Taking the logarithm of both sides of inequality (38), we get

$$
-n\left(\sum_{k=1}^{m} \log \left[1-\frac{\varphi_{k}}{n}\right]\right)<\sum_{k=1}^{m} \varphi_{k} .
$$

But for each $k, k=1, \ldots, m$, we have

$$
-n\left(\log \left[1-\frac{\varphi_{k}}{n}\right]+\frac{\varphi_{k}}{n}\right)=n\left(\left[1-\frac{\varphi_{k}}{n}\right]-1-\log \left[1-\frac{\varphi_{k}}{n}\right]\right) \geq 0,
$$

since $\log \alpha \leq \alpha-1$ with equality if and only if $\alpha=1$. This contradicts (39) and proves inequality (31). Note that, as $n \rightarrow \infty$, the equality holds. The proof is complete.

As a numerical example, in Figure 2 we plot the values of both functions ${ }_{0} F_{1}\left(n ; X^{H} X\right)$ and ${ }_{0} F_{0}\left(X^{H} X / n\right)$ vs $k(0 \leq k \leq 20)$, where we have chosen $m=2, n=10$ and the eigenvalues of $X^{H} X$ are 1 and 0.75 . It can be seen that the derived inequality is quite tight and the series expansions are converging quickly.

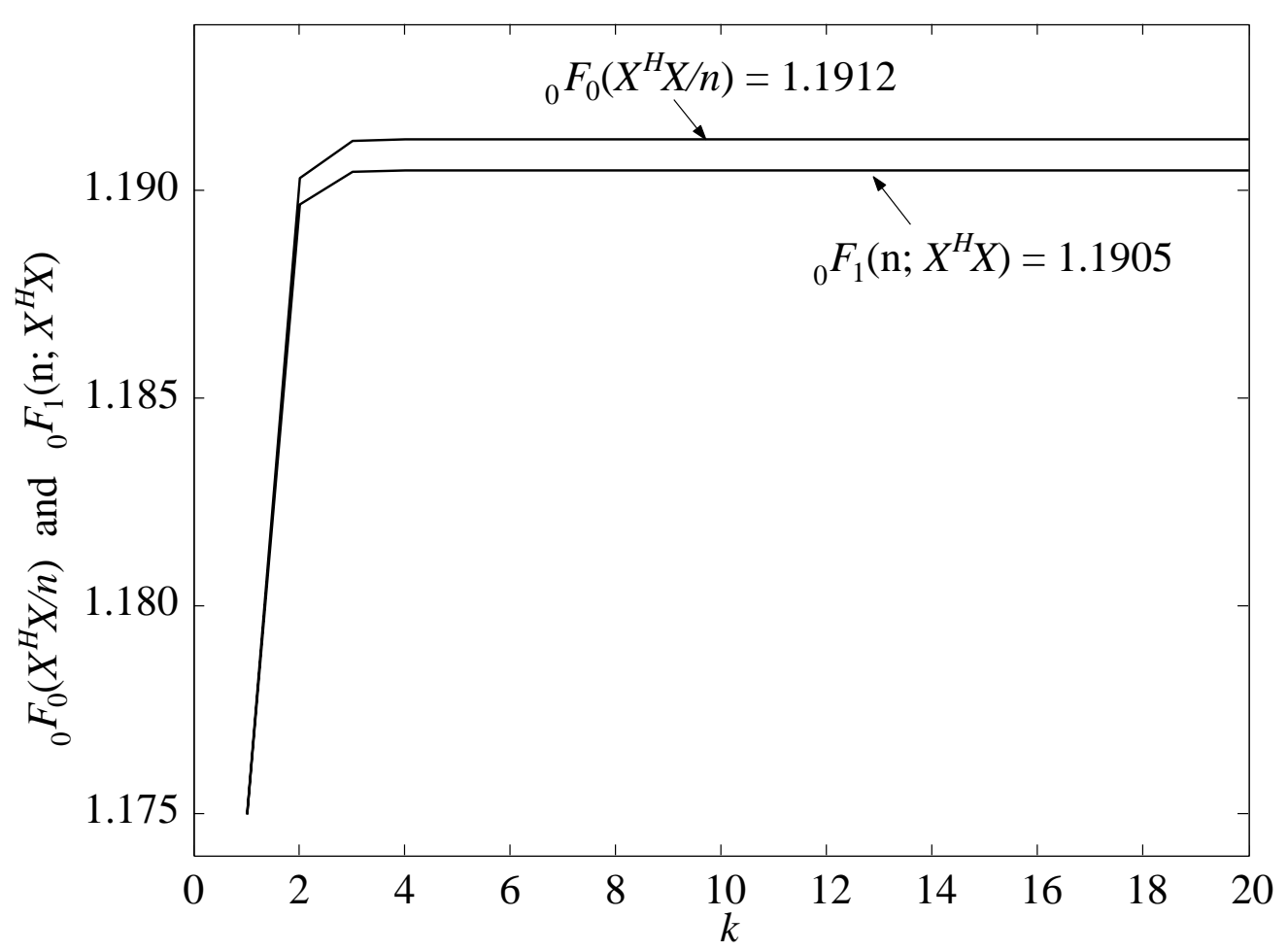

Figure 2: The functions ${ }_{0} F_{1}\left(n ; X^{H} X\right)$ and ${ }_{0} F_{0}\left(X^{H} X / n\right)$ vs $k$, where $m=2, n=10$ and the eigenvalues of $X^{H} X$ are 1 and 0.75 .

The joint eigenvalue density of a complex noncentral Wishart matrix can be expressed by a bounded density function, which is given by the following theorem. 
Theorem 7 Let $\mathrm{W} \sim \mathcal{C} W_{m}(n, \Sigma, \Omega)$ with $n \geq m$. Then $\mathrm{W}$ is an $m \times m$ positive definite Hermitian matrix. The joint density of the eigenvalues, $\lambda_{1}>\cdots>\lambda_{m}>0$, of $\mathrm{W}$ satisfies the inequality

$$
f(\Lambda) \leq \frac{\pi^{m(m-1)}(\operatorname{det} \Sigma)^{-n}}{\mathcal{C} \Gamma_{m}(m) \mathcal{C} \Gamma_{m}(n)} \operatorname{etr}(-\Omega) \prod_{k=1}^{m} \lambda_{k}^{n-m} \prod_{k<l}^{m}\left(\lambda_{k}-\lambda_{l}\right)^{2}{ }_{0} F_{0}^{(m)}(-\Psi, \Lambda),
$$

where $\Lambda=\operatorname{diag}\left(\lambda_{1}, \ldots, \lambda_{m}\right)$, the diagonal elements of $\Psi, \psi_{1}, \ldots, \psi_{m}$, are the eigenvalues of the matrix $\left(\Sigma^{-1}-\Omega \Sigma^{-1} / n\right)$, and $\Omega=\Sigma^{-1} M^{H} M$.

Proof. From Proposition 1 we obtain

$$
\begin{aligned}
f(\Lambda)= & \frac{\pi^{m(m-1)}}{\mathcal{C} \Gamma_{m}(m)} \prod_{k<l}^{m}\left(\lambda_{k}-\lambda_{l}\right)^{2} \int_{U(m)} f\left(E \Lambda E^{H}\right)(d E) \\
= & \frac{\pi^{m(m-1)}(\operatorname{det} \Sigma)^{-n}}{\mathcal{C} \Gamma_{m}(m) \mathcal{C} \Gamma_{m}(n)} \operatorname{etr}(-\Omega) \prod_{k=1}^{m} \lambda_{k}^{n-m} \prod_{k<l}^{m}\left(\lambda_{k}-\lambda_{l}\right)^{2} \\
& \times \int_{U(m)} \operatorname{etr}\left(-\Sigma^{-1} E \Lambda E^{H}\right)_{0} F_{1}\left(n ; \Omega \Sigma^{-1} E \Lambda E^{H}\right)(d E) \\
\leq & \frac{\pi^{m(m-1)}(\operatorname{det} \Sigma)^{-n}}{\mathcal{C} \Gamma_{m}(m) \mathcal{C} \Gamma_{m}(n)} \operatorname{etr}(-\Omega) \prod_{k=1}^{m} \lambda_{k}^{n-m} \prod_{k<l}^{m}\left(\lambda_{k}-\lambda_{l}\right)^{2} \\
& \left.\times \int_{U(m)} \operatorname{etr}\left(-\Sigma^{-1} E \Lambda E^{H}\right)_{0} F_{0}\left(\Omega \Sigma^{-1} E \Lambda E^{H} / n\right)(d E) \quad \text { (by Lemma } \mathbb{1}\right) \\
\leq & \frac{\pi^{m(m-1)}(\operatorname{det} \Sigma)^{-n}}{\mathcal{C} \Gamma_{m}(m) \mathcal{C} \Gamma_{m}(n)} \operatorname{etr}(-\Omega) \prod_{k=1}^{m} \lambda_{k}^{n-m} \prod_{k<l}^{m}\left(\lambda_{k}-\lambda_{l}\right)^{2} \\
& \times \int_{U(m)} \operatorname{etr}\left(-\left(\Sigma^{-1}-\Omega \Sigma^{-1} / n\right) E \Lambda E^{H}\right)(d E) \\
\leq & \frac{\pi^{m(m-1)}(\operatorname{det} \Sigma)^{-n}}{\mathcal{C} \Gamma_{m}(m) \mathcal{C} \Gamma_{m}(n)} \operatorname{etr}(-\Omega) \prod_{k=1}^{m} \lambda_{k}^{n-m} \prod_{k<l}^{m}\left(\lambda_{k}-\lambda_{l}\right)^{2}{ }_{0} F_{0}^{(m)}(-\Psi, \Lambda) .
\end{aligned}
$$

The result follows from equation (6).

Note that the function ${ }_{0} F_{0}^{(m)}(-\Psi, \Lambda)$ can be written [21] as

$$
{ }_{0} F_{0}^{(m)}(-\Psi, \Lambda)=\frac{\mathcal{C} \Gamma_{m}(m) \operatorname{det}\left[\left(\exp \left(-\psi_{i} \lambda_{j}\right)\right)\right]}{\pi^{m(m-1) / 2} \prod_{k<l}^{m}\left(\lambda_{k}-\lambda_{l}\right) \prod_{k<l}^{m}\left(\psi_{l}-\psi_{k}\right)} .
$$

The following theorem expresses the capacity in terms of the unordered eigenvalue density.

Theorem 8 Consider a Rician channel, i.e., $\mathrm{H} \sim \mathcal{C} N\left(M, I_{n_{r}} \otimes \Sigma\right)$, with $n_{r} \geq n_{t}$. If the input power is constrained by $\rho$, then using the unordered eigenvalue density we can bound the capacity $C$ as

$$
C \leq n_{t} \mathcal{E}_{\lambda_{1}}\left[\log \left(1+\left(\rho / n_{t}\right) \lambda_{1}\right)\right] .
$$

Moreover, the density $f\left(\lambda_{1}\right)$ satisfies the inequality

$$
\begin{aligned}
f\left(\lambda_{1}\right) \leq \frac{\pi^{n_{t}\left(n_{t}-1\right) / 2}(\operatorname{det} \Sigma)^{-n_{r}} \operatorname{etr}(-\Omega)}{n_{t} ! \mathcal{C} \Gamma_{n_{t}}\left(n_{r}\right) \prod_{k<l}^{n_{t}}\left(\psi_{l}-\psi_{k}\right)} & \int\left\{\widetilde{\sum_{i}}(-1)^{\operatorname{per}\left(i_{1}, \ldots, i_{n_{t}}\right)} \exp \left(\sum_{j=1}^{n_{t}}-\lambda_{j} \psi_{i_{j}}\right)\right\} \\
& \times\left\{\widetilde{\sum_{k}}(-1)^{\operatorname{per}\left(k_{1}, \ldots, k_{n_{t}}\right)} \prod_{l=1}^{n_{t}} \lambda_{l}^{n_{r}-n_{t}+k_{l}}\right\} \bigwedge_{k=2}^{n_{t}} d \lambda_{k},
\end{aligned}
$$


where $\widetilde{\sum}_{i}$ denotes summation over all permutations $\left(i_{1}, \ldots, i_{n_{t}}\right)$ of $\left(1, \ldots, n_{t}\right), \widetilde{\sum_{k}}$ denotes summation over all permutations $\left(k_{1}, \ldots, k_{n_{t}}\right)$ of $\left(0, \ldots, n_{t}-1\right)$ and $\left\{\operatorname{per}\left(k_{1}, \ldots, k_{n_{t}}\right)\right\}$ is 0 or 1 depending on the permutation being even or odd. Similarly for $\left\{\operatorname{per}\left(i_{1}, \ldots, i_{n_{t}}\right)\right\}$.

Proof. From equation (30), $C$ can be written as

$$
C=\sum_{k=1}^{n_{t}} \mathcal{E}_{\lambda_{k}}\left[\log \left(1+\frac{\rho}{n_{t}} \lambda_{k}\right)\right]=n_{t} \mathcal{E}_{\lambda_{1}}\left[\log \left(1+\frac{\rho}{n_{t}} \lambda_{1}\right)\right]
$$

where the expectation is with respect to the unordered eigenvalue density $f\left(\lambda_{1}\right)$. The bounded unordered eigenvalue density (43) is obtained by substituting (41) in (40) and integrating with respect to $\lambda_{2}, \ldots, \lambda_{n_{t}}$, i.e.,

$$
f\left(\lambda_{1}\right) \leq \frac{\pi^{n_{t}\left(n_{t}-1\right) / 2}(\operatorname{det} \Sigma)^{-n_{r}} \operatorname{etr}(-\Omega)}{n_{t} ! \mathcal{C} \Gamma_{n_{t}}\left(n_{r}\right) \prod_{k<l}^{n_{t}}\left(\psi_{l}-\psi_{k}\right)} \int \operatorname{det}\left[\left(\exp \left(-\psi_{i} \lambda_{j}\right)\right)\right] \prod_{k<l}^{n_{t}}\left(\lambda_{k}-\lambda_{l}\right) \prod_{k=1}^{n_{t}} \lambda_{k}^{n_{r}-n_{t}} \bigwedge_{k=2}^{n_{t}} d \lambda_{k}
$$

The integrand in equation (45) can be written as

$$
\begin{aligned}
& \operatorname{det}\left[\left(\exp \left(-\psi_{i} \lambda_{j}\right)\right)\right] \prod_{k<l}^{n_{t}}\left(\lambda_{k}-\lambda_{l}\right) \prod_{k=1}^{n_{t}} \lambda_{k}^{n_{r}-n_{t}} \\
& =\operatorname{det}\left[\begin{array}{ccc}
e^{-\psi_{1} \lambda_{1}} & \ldots & e^{-\psi_{1} \lambda_{n_{t}}} \\
e^{-\psi_{2} \lambda_{1}} & \ldots & e^{-\psi_{2} \lambda_{n_{t}}} \\
\vdots & \vdots & \vdots \\
e^{-\psi_{n_{t} \lambda_{1}}} & \ldots & e^{-\psi_{n_{t} \lambda_{n_{t}}}}
\end{array}\right] \operatorname{det}\left[\begin{array}{ccc}
1 & \ldots & 1 \\
\lambda_{1} & \ldots & \lambda_{n_{t}} \\
\vdots & \vdots & \vdots \\
\lambda_{1}^{n_{t}-1} & \ldots & \lambda_{n_{t}}^{n_{t}-1}
\end{array}\right] \prod_{k=1}^{n_{t}} \lambda_{k}^{n_{r}-n_{t}} \\
& =\operatorname{det}\left[\begin{array}{ccc}
e^{-\psi_{1} \lambda_{1}} & \ldots & e^{-\psi_{1} \lambda_{n_{t}}} \\
\vdots & \vdots & \vdots \\
e^{-\psi_{n_{t}} \lambda_{1}} & \ldots & e^{-\psi_{n_{t}} \lambda_{n_{t}}}
\end{array}\right] \operatorname{det}\left[\begin{array}{ccc}
\lambda_{1}^{n_{r}-n_{t}} & \ldots & \lambda_{n_{t}}^{n_{r}-n_{t}} \\
\vdots & \vdots & \vdots \\
\lambda_{1}^{n_{r}-1} & \ldots & \lambda_{n_{t}}^{n_{r}-1}
\end{array}\right] \\
& =\left\{\widetilde{\sum_{i}}(-1)^{\operatorname{per}\left(i_{1}, \ldots, i_{n_{t}}\right)} \exp \left(\sum_{j=1}^{n_{t}}-\psi_{i_{j}} \lambda_{j}\right)\right\}\left\{\widetilde{\sum_{k}}(-1)^{\operatorname{per}\left(k_{1}, \ldots, k_{n_{t}}\right)} \prod_{l=1}^{n_{t}} \lambda_{l}^{n_{r}-n_{t}+k_{l}}\right\} \text {. }
\end{aligned}
$$

The result follows.

\section{Computation of the Capacities}

In this subsection, we find numerically an upper bound for an $n_{r} \times 2$ Rician channel capacity. Thus, we have a two-input $\left(n_{t}=2\right), n_{r}$-output communication system operating over a Rician fading environment (typical satellite communication environment). Let $n_{t}=2$ and $\Psi=\operatorname{diag}\left(\psi_{1}, \psi_{2}\right)$. Then we have [21]

$$
{ }_{0} F_{0}^{(2)}(-\Psi, \Lambda)=\frac{1}{\left(\psi_{2}-\psi_{1}\right)\left(\lambda_{1}-\lambda_{2}\right)}\left[e^{-\psi_{1} \lambda_{1}-\psi_{2} \lambda_{2}}-e^{-\psi_{1} \lambda_{2}-\psi_{2} \lambda_{1}}\right]
$$

The following theorem gives an upper bound for the Rician channel capacity for an $n_{r} \times 2$ matrix. 
Theorem 9 Consider a two-input Rician channel, i.e., $\mathrm{H} \sim \mathcal{C} N\left(M, I_{n_{r}} \otimes \Sigma\right)$, with $n_{r} \geq 2$. If the input power is constrained by $\rho$, then the capacity $C$ satisfies the inequality

$$
\begin{aligned}
C \leq & \frac{(\operatorname{det} \Sigma)^{-n_{r}} \operatorname{etr}(-\Omega)}{\left(\psi_{2}-\psi_{1}\right)}\left[\frac{\psi_{2}^{-n_{r}+1}}{\Gamma\left(n_{r}\right)} \int_{0}^{\infty} \log \left[1+\frac{\rho}{2} \lambda_{1}\right] \lambda_{1}^{n_{r}-1} e^{-\psi_{1} \lambda_{1}} d \lambda_{1}\right. \\
& -\frac{\psi_{1}^{-n_{r}+1}}{\Gamma\left(n_{r}\right)} \int_{0}^{\infty} \log \left[1+\frac{\rho}{2} \lambda_{1}\right] \lambda_{1}^{n_{r}-1} e^{-\psi_{2} \lambda_{1}} d \lambda_{1} \\
& -\frac{\psi_{2}^{-n_{r}}}{\Gamma\left(n_{r}-1\right)} \int_{0}^{\infty} \log \left[1+\frac{\rho}{2} \lambda_{1}\right] \lambda_{1}^{n_{r}-2} e^{-\psi_{1} \lambda_{1}} d \lambda_{1} \\
& \left.+\frac{\psi_{1}^{-n_{r}}}{\Gamma\left(n_{r}-1\right)} \int_{0}^{\infty} \log \left[1+\frac{\rho}{2} \lambda_{1}\right] \lambda_{1}^{n_{r}-2} e^{-\psi_{2} \lambda_{1}} d \lambda_{1}\right],
\end{aligned}
$$

where $\lambda_{1}$ is an unordered eigenvalue of $W=H^{H} H$, and $\psi_{1}$ and $\psi_{2}$ are the eigenvalues of $\left(\Sigma^{-1}-\Omega \Sigma^{-1} / n_{r}\right)$.

Proof. Using equation (46), the unordered eigenvalue density of $W$ satisfies the inequality

$$
f\left(\lambda_{1}, \lambda_{2}\right) \leq \frac{(\operatorname{det} \Sigma)^{-n_{r}} \operatorname{etr}(-\Omega)\left(\lambda_{1} \lambda_{2}\right)^{n_{r}-2}\left(\lambda_{1}-\lambda_{2}\right)}{2\left(\psi_{2}-\psi_{1}\right) \Gamma\left(n_{r}\right) \Gamma\left(n_{r}-1\right)}\left[e^{-\psi_{1} \lambda_{1}-\psi_{2} \lambda_{2}}-e^{-\psi_{1} \lambda_{2}-\psi_{2} \lambda_{1}}\right] .
$$

Now, integrating with respect to $\lambda_{2}$ and noting that

$$
\int_{0}^{\infty} x^{a-1} e^{-x / b} d x=\Gamma(a) b^{a},
$$

we obtain the density of $f\left(\lambda_{1}\right)$. Thus we have

$$
\begin{aligned}
f\left(\lambda_{1}\right) \leq & \frac{(\operatorname{det} \Sigma)^{-n_{r}} \operatorname{etr}(-\Omega)}{2\left(\psi_{2}-\psi_{1}\right)} \\
& \times\left\{\frac{\lambda_{1}^{n_{r}-1} e^{-\psi_{1} \lambda_{1}}}{\Gamma\left(n_{r}\right) \psi_{2}^{n_{r}-1}}-\frac{\lambda_{1}^{n_{r}-1} e^{-\psi_{2} \lambda_{1}}}{\Gamma\left(n_{r}\right) \psi_{1}^{n_{r}-1}}-\frac{\lambda_{1}^{n_{r}-2} e^{-\psi_{1} \lambda_{1}}}{\Gamma\left(n_{r}-1\right) \psi_{2}^{n_{r}}}+\frac{\lambda_{1}^{n_{r}-2} e^{-\psi_{2} \lambda_{1}}}{\Gamma\left(n_{r}-1\right) \psi_{1}^{n_{r}}}\right\} .
\end{aligned}
$$

Finally, evaluating equation (44) with $f\left(\lambda_{1}\right)$ gives inequality (47).

Figure 3 shows the capacity in nats ${ }^{6}$ vs SNR for $n_{r}=2$ and 4 , and $n_{t}=2$, where the solid lines represent the upper bounds given in (47) and the dashed lines represent the simulation results. It can be seen that the derived capacity upper bound is quite tight with the simulation results for the entire range of SNRs, and this illustrates the accuracy of the bound.

Table 1 lists the capacity upper bound in nats for an $n_{r} \times 2$ Rician fading channel matrix. Note that each column represents different levels of input power or signal-to-noise ratio (SNR) in dB. Figure 4 shows the capacity (upper bound) in nats vs $n_{r}$ for different levels of input power. From the table and figures we note that the capacity is increasing with increasing $n_{r}$ and SNR. Moreover, the Rician channel capacity is increasing compared to an uncorrelated Rayleigh channel capacity, see [26, Table 2]. Note that here we have assumed an uncorrelated Rician channel $\mathrm{H} \sim \mathcal{C} N\left(M, I_{n_{r}} \otimes \Sigma\right)$, where the covariance matrix and the mean matrix are

$$
\Sigma=\left[\begin{array}{ll}
1 & 0 \\
0 & 1
\end{array}\right] \text { and } M=\left[\begin{array}{cc}
0.2+0.2 i & 0.2+0.2 i \\
0.2+0.2 i & 0.2+0.2 i
\end{array}\right]
$$

respectively.

\footnotetext{
${ }^{6}$ In equation (47), if we use $\log _{e}$ then the capacity is measured in nats. If we use $\log _{2}$ then the capacity is measured in bits. Thus, one nat is equal to $1 / \log _{e} 2 \mathrm{bits} / \mathrm{sec} / \mathrm{Hz}(e=2.718 \ldots)$.
} 
Table 1: The capacity upper bound in nats for a two-input, $n_{r}$-output communication system operating over a Rician fading channel, where $\rho$ is signal-to-noise ratio in $\mathrm{dB}$.

\begin{tabular}{c|c|c|c|c|c|c|c|c}
\hline & \multicolumn{7}{|c}{$\rho \mathrm{in} \mathrm{dB}$} \\
\hline$n_{r}$ & $0 \mathrm{~dB}$ & $5 \mathrm{~dB}$ & $10 \mathrm{~dB}$ & $15 \mathrm{~dB}$ & $20 \mathrm{~dB}$ & $25 \mathrm{~dB}$ & $30 \mathrm{~dB}$ & $35 \mathrm{~dB}$ \\
\hline 2 & 1.2739 & 2.4644 & 4.0886 & 6.0327 & 8.1639 & 10.3803 & 12.6284 & 14.8873 \\
4 & 2.2125 & 3.9525 & 6.0789 & 8.3996 & 10.7980 & 13.2235 & 15.6580 & 18.0953 \\
6 & 2.9542 & 4.9766 & 7.2964 & 9.7406 & 12.2289 & 14.7317 & 17.2392 & 19.7482 \\
8 & 3.5817 & 5.7894 & 8.2351 & 10.7721 & 13.3401 & 15.9181 & 18.4994 & 21.0817 \\
10 & 4.1389 & 6.4900 & 9.0396 & 11.6620 & 14.3086 & 16.9629 & 19.6198 & 22.2774 \\
12 & 4.6512 & 7.1248 & 9.7699 & 12.4758 & 15.2018 & 17.9342 & 20.6686 & 23.4037 \\
14 & 5.1338 & 7.7191 & 10.4560 & 13.2457 & 16.0527 & 18.8652 & 21.6794 & 24.4942 \\
16 & 5.5967 & 8.2878 & 11.1155 & 13.9901 & 16.8800 & 19.7748 & 22.6711 & 25.5679 \\
18 & 6.0470 & 8.8406 & 11.7593 & 14.7206 & 17.6955 & 20.6749 & 23.6557 & 26.6369 \\
20 & 6.4895 & 9.3843 & 12.3950 & 15.4448 & 18.5071 & 21.5735 & 24.6411 & 27.7092 \\
\hline
\end{tabular}

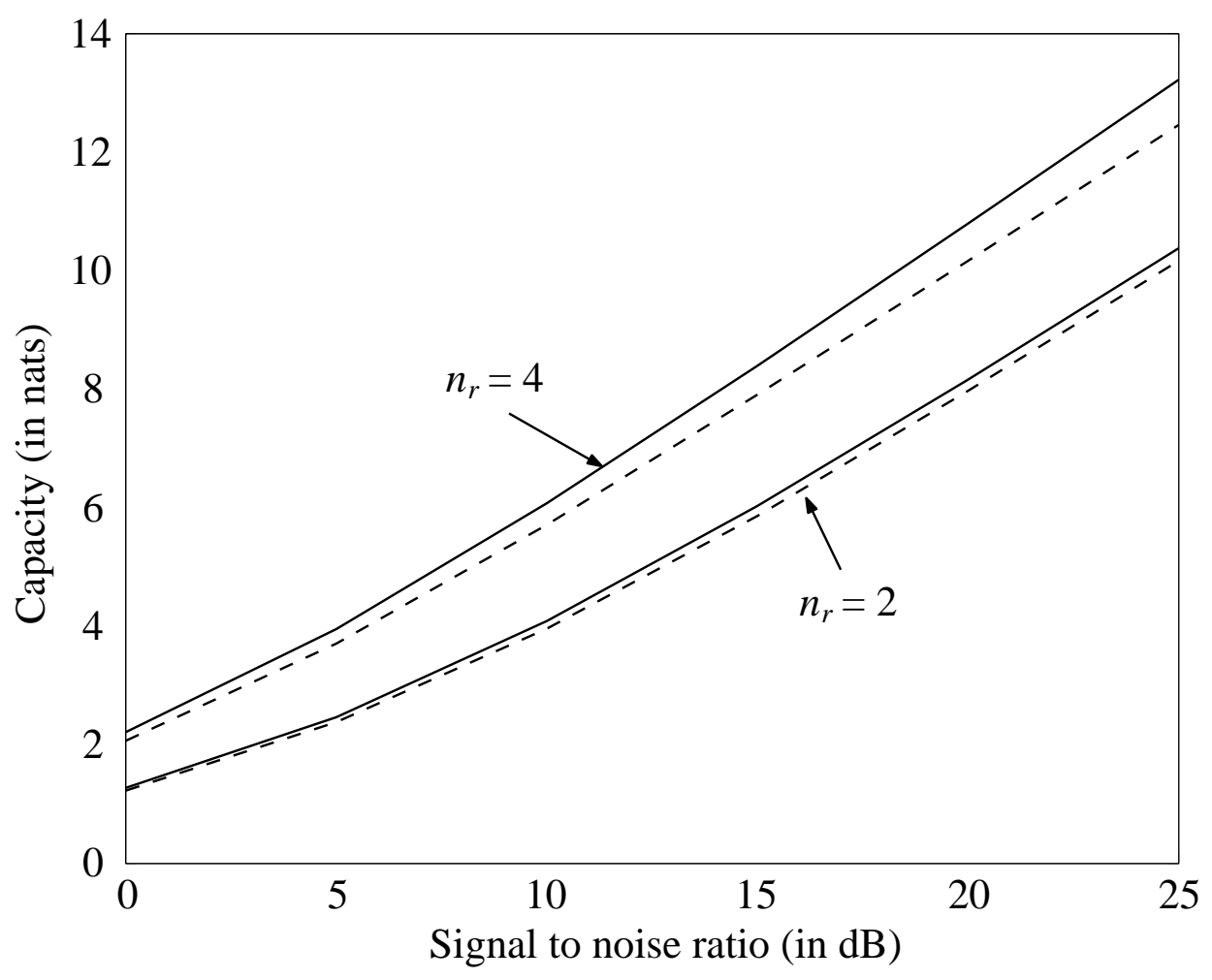

Figure 3: Capacity vs SNR for $n_{t}=2$ and $n_{r}=2$ and 4, i.e., $\mathrm{H}$ is an $n_{r} \times 2$ Rician fading channel matrix. The solid lines represent the upper bounds given in (47) and the dashed lines represent the simulation results. 


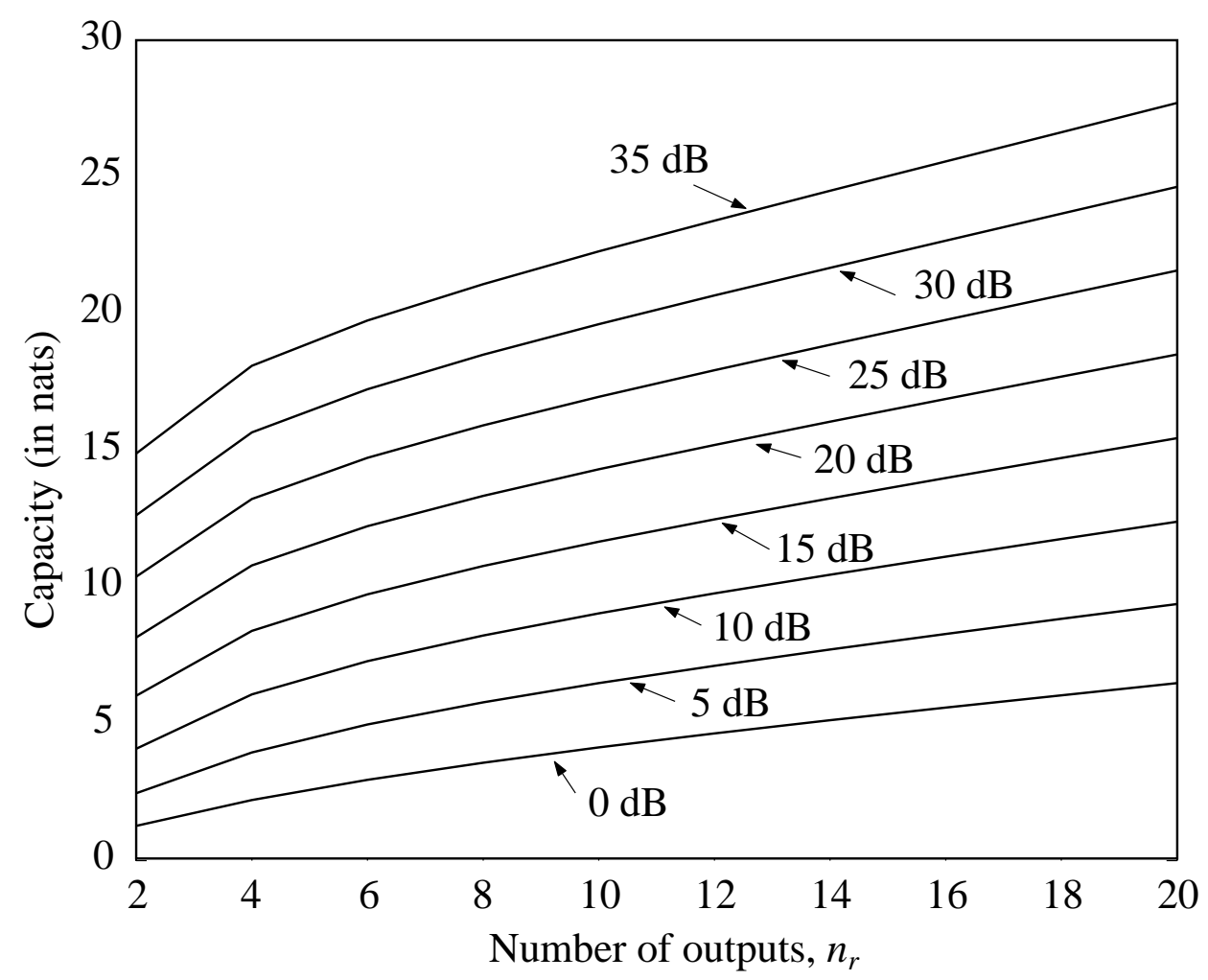

Figure 4: Capacity upper bound vs number of outputs for $\mathrm{SNR}=0,5,10,15,20,25,30,35 \mathrm{~dB}$. Note that $\mathrm{H}$ is an $n_{r} \times 2$ Rician fading channel matrix.

Next, we consider the spatial correlation effects; hence, in this case, the covariance matrix is

$$
\Sigma=\left[\begin{array}{ll}
1 & \varrho \\
\varrho & 1
\end{array}\right] .
$$

Note also that the off-diagonal element of $\Sigma$ gives the correlation between the channel coefficient from different transmitter antennas to a single receiver antenna, i.e.,

$$
\mathcal{E}\left\{h_{i j} h_{k l}^{*}\right\}= \begin{cases}\varrho & i \neq k=1,2, j=l=1, \ldots, n_{r}, \\ 0 & \text { otherwise }\end{cases}
$$

This off-diagonal element is called the channel correlation coefficient or correlation coefficient. Figure 5 shows the capacity upper bound in nats vs SNR for the correlation coefficient $\varrho=0.99$ and $n_{t}=n_{r}=2$. Note that $C_{u}$ and $C_{c}$ denote the capacity of uncorrelated and spatially correlated (at the transmitter end) channels, respectively. It is clear from Figure 5 that the capacity is degraded by $33 \%$ due to correlation compared to the uncorrelated case at SNR $=15 \mathrm{~dB}$. Figure 6 shows the capacity upper bound in nats vs the correlation coefficient for SNR $=25 \mathrm{~dB}$ and $n_{t}=2$ and $n_{r}=2,4$. From these figures we note the following: (i) the capacity is decreasing with increasing channel correlation, and (ii) the capacity is increasing with increasing $n_{r}$ and SNR.

\section{Conclusion}

In this paper, the densities of the largest, smallest and joint eigenvalues of a complex noncentral Wishart matrix are derived for an arbitrary mean and covariance matrix. These densities play an 


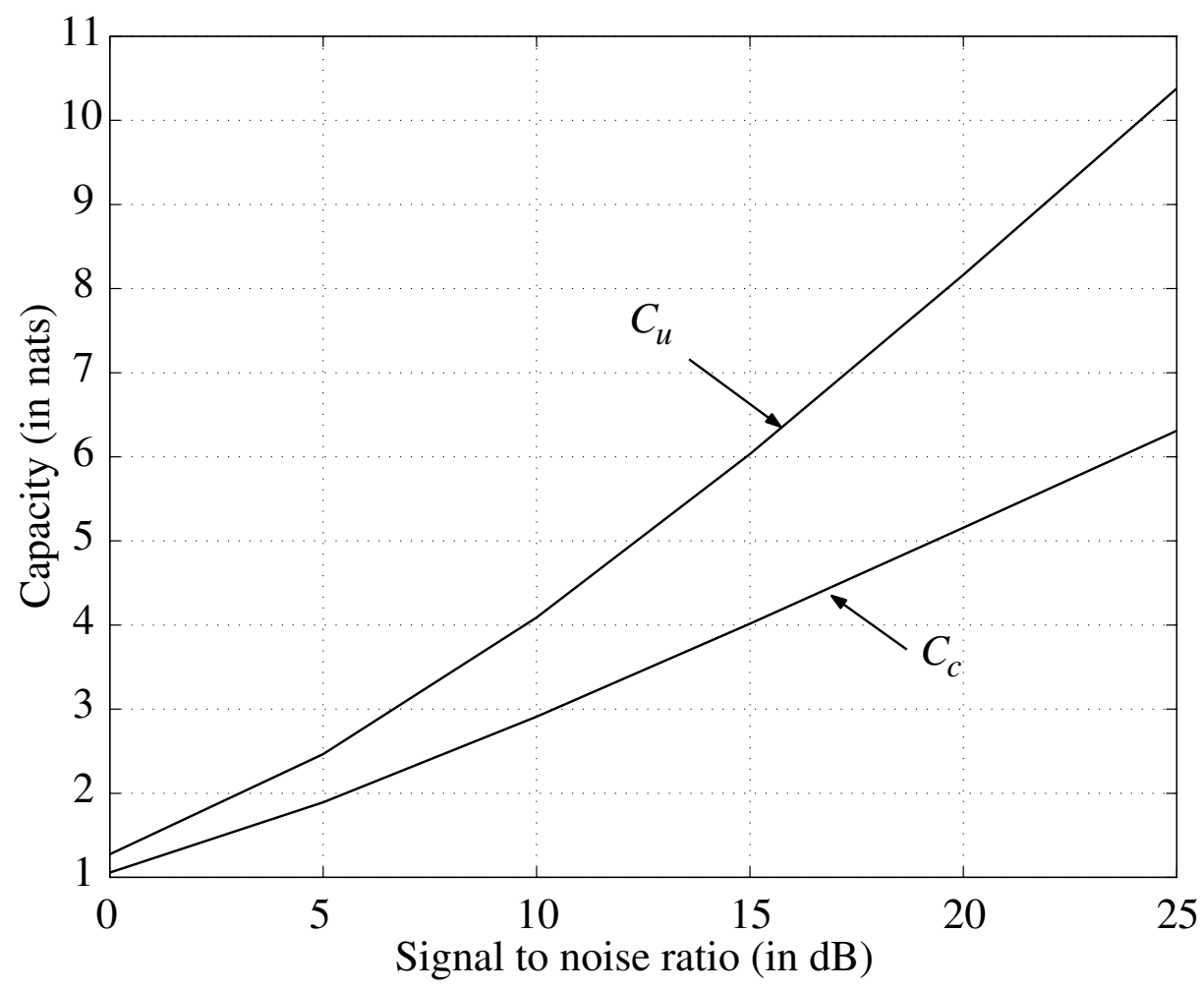

Figure 5: Capacity upper bound vs SNR for correlation coefficient $\varrho=0.99, n_{t}=2$, and $n_{r}=2$, i.e., $\mathrm{H}$ is a $2 \times 2$ correlated Rician fading channel matrix. Note that $C_{u}$ and $C_{c}$ denote the capacity of uncorrelated and spatially correlated (at the transmitter end) channels, respectively.

important role in information theory, numerical analysis and statistical hypothesis testing. We show that the joint eigenvalue density of a complex noncentral Wishart matrix can be expressed by a easily computable bounded density function. Furthermore, A single unordered eigenvalue density of complex noncentral Wishart matrix also derived. Using these densities the most important information-theoretic measure, the so-called ergodic channel capacity formulas for MIMO Rician channel are derived. Specifically, the exact and easily computable tight upper bound formulas for ergodic capacity is given for both spatially correlated and uncorrelated MIMO Rician channels. Numerical results are also given, which show how the channel correlation degrades the capacity of the communication system. For example at $\mathrm{SNR}=15 \mathrm{~dB}$ and correlation coefficient $\varrho=0.99$, the capacity of $2 \times 2$ Rician channel is degraded by $33 \%$ due to correlation compared to the uncorrelated case, see Figure 5 .

\section{Acknowledgement}

The authors thanks the referees for their comments and corrections which improved this paper.

\section{References}

[1] R. B. Ash, Information Theory, Dover, New York, 1965.

[2] M. Chiani, M. Z. Win and A. Zanella, "On the capacity of spatially correlated MIMO RayleighFading channels," IEEE Trans. on Information Theory, Vol. 49, pp. 2363-2371, Oct. 2003. 


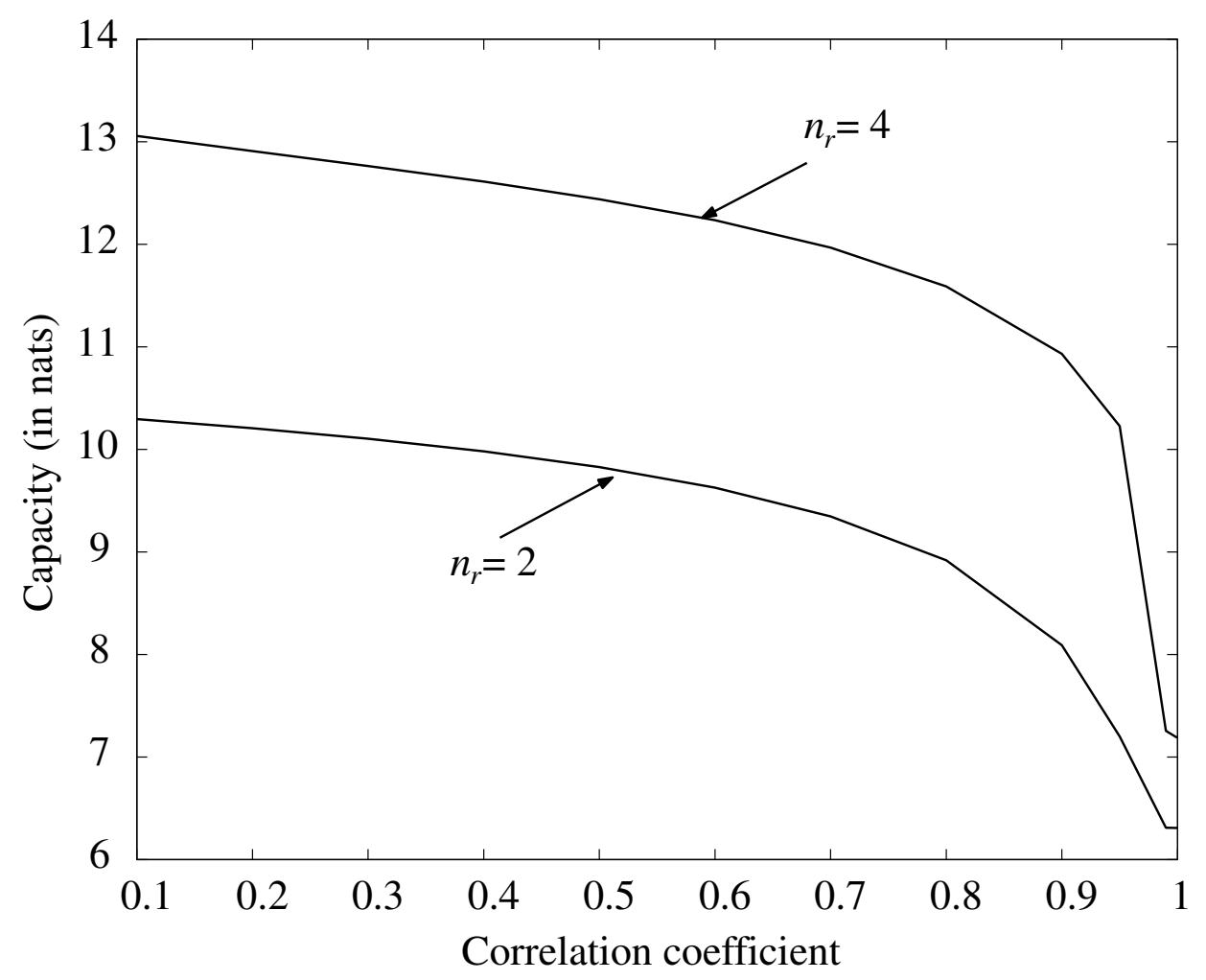

Figure 6: Capacity upper bound vs correlation coefficient for SNR $=25 \mathrm{~dB}, n_{t}=2$, and $n_{r}=2,4$, i.e., $\mathrm{H}$ is an $n_{r} \times 2$ correlated Rician fading channel matrix.

[3] C. N. Chuah, D. Tse, J. M. Kahn and R. A. Valenzuela, "Capacity scaling in MIMO wireless systems under correlated fading," IEEE Trans. on Information Theory, Vol. 48, pp. 637-650, Mar. 2002.

[4] Y. Chikuse and A. W. Davis, "Some properties of invariant polynomials with matrix arguments and their applications in econometrics," Ann. Inst. Statist. Math., Vol. 38, pp. 109-122, 1986.

[5] A. G. Constantine, "Some noncentral distribution problems in multivariate analysis," Ann. Math. Statist., Vol. 34, pp. 1270-1285, 1963.

[6] A. W. Davis, "Invariant polynomials with two matrix arguments extending the zonal polynomials: Applications to multivariate distribution theory," Ann. Inst. Statist. Math., Vol. 31, pp. 465-485, 1979.

[7] A. W. Davis, "Invariant polynomials with two matrix arguments extending the zonal polynomials," Multivariate Analysis- V (ed. P. K. Krishnaiah), North-Holland, Amsterdam, pp. 287-299, 1980.

[8] A. Edelman, Eigenvalues and Condition Numbers of Random Matrices, Ph.D. Dissertation, MIT, Cambridge, MA, May 1989.

[9] J. G. Foschini, "Layered space-time architecture for wireless communication in a fading environment when using multi-element antennas," Bell Labs Technical J., pp. 41-59, Autumn 1996. 
[10] J. G. Foschini and M. J. Gans, "On limits of wireless communications in a fading environment when using multiple antennas," Wireless Personal Communications, Vol. 6, pp. 311-335, Mar. 1998.

[11] N. R. Goodman, "Statistical analysis based on a certain multivariate complex Gaussian distribution (An introduction)," Ann. Math. Statist., Vol. 34, pp. 152-177, 1963.

[12] A. T. James, "Normal multivariate analysis and the orthogonal group," Ann. Math. Statist., Vol. 25, pp. 40-75, 1954.

[13] A. T. James, "Distributions of matrix variate and latent roots derived from normal samples," Ann. Math. Statist., Vol. 35, pp. 475-501, 1964.

[14] S. Jayaweera and H. Poor, "On the capacity of multi-antenna systems in the presence of Rician fading," in Proc. IEEE Veh. Tech. Conf., Vancouver, pp. 1963-1967, Sep. 2002.

[15] M. Kang and M. -S. Alouini, "Performance analysis of MIMO MRC systems over Rician fading channels," in Proc. IEEE Veh. Tech. Conf., Vancouver, pp. 869-873, Sep. 2002.

[16] M. Kang and M. -S. Alouini, "On the capacity of MIMO Rician channel," in Proc. 40th Annual Allerton Conference on Communication, Control, and Computing, Monticello, Oct. 2002.

[17] M. Kang and M. -S. Alouini, "Impact of correlation on the capacity of MIMO channels," in Proc. IEEE Int. Conf. Commun., Anchorage, pp. 2623-2627, May 2003.

[18] M. Kang, L. Yang and M. -S. Alouini, "Capacity of MIMO Rician channels with multiple correlated Rayleigh co-channel interferers," in Proc. IEEE Global Telecom., San Francisco, pp. 1119-1123, Dec. 2003.

[19] M. Kang and M. -S. Alouini, "Largest eigenvalue of complex Wishart matrices and performance analysis of MIMO MRC systems," IEEE J. Select. Areas Commun., Vol. 21, pp. 406-417, Apr. 2003.

[20] C. G. Khatri, "On certain distribution problems based on positive definite quadratic functions in normal vectors," Ann. Math. Statist., Vol. 37, pp. 468-479, 1966.

[21] C. G. Khatri, "Non-central distributions of $i$ th largest characteristic roots of three matrices concerning complex multivariate normal populations," Ann. Inst. Statist. Math., Vol. 21, pp. 23-32, 1969.

[22] M. L Mehta, Random Matrices, 2nd ed., Academic Press, New York, 1991.

[23] K. S. Miller, Complex Stochastic Processes: An Introduction to Theory and Application, Addison-Wesley, New York, 1974.

[24] R. J. Muirhead, Aspects of Multivariate Statistical Theory, Wiley, New York, 1982.

[25] T. Ratnarajah, R. Vaillancourt and M. Alvo, "Eigenvalues and condition numbers of complex random matrices," SIAM Journal on Matrix Analysis and Applications, in press (Apr. 2004).

[26] T. Ratnarajah, R. Vaillancourt and M. Alvo, "Complex random matrices and Rayleigh channel capacity," Communications in Information \& Systems, Vol. 3, No. 2, pp. 119-138, Oct. 2003

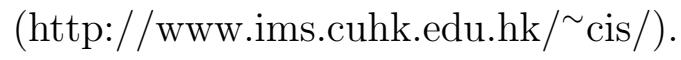


[27] T. Ratnarajah, R. Vaillancourt and M. Alvo, "Jacobians and hypergeometric functions in complex multivariate analysis," Canadian Applied Mathematics Quarterly, in press (Jul. 2003).

[28] T. Ratnarajah and R. Vaillancourt, "Quadratic forms on complex random matrices and multiple-antenna channel capacity," In Proc. The Twelfth Annual Workshop on Adaptive Sensor Array Processing, MIT Lincoln Laboratory, Massachusetts, Mar. 16-18, 2004.

[29] T. Ratnarajah and R. Vaillancourt, "Quadratic forms on complex random matrices and channel capacity," In Proc. IEEE International Conference on Acoustics, Speech, and Signal Processing, Montreal, Quebec, Vol. 4, pp. 385-388, May 17-21, 2004.

[30] M. Sellathurai and G. Foschini, "A stratified diagonal layered space-time architecture: Information theoretic and signal processing aspects," IEEE Trans. on Signal Processing, Vol. 51, pp. 2943-2954, Nov. 2003.

[31] H. Shin and J. H. Lee, "Capacity of multiple-antenna fading channels: spatial fading correlation, double scattering, and keyhole," IEEE Trans. on Information Theory, Vol. 49, pp. 2636-2647, Oct. 2003.

[32] D. S. Shiu, G. F. Foschini, M. G. Gans and J. M. Kahn, "Fading correlation and its effect on the capacity of multielement antenna systems," IEEE Trans. on Communications, Vol. 48, pp. 502-513, Mar. 2000.

[33] T. Sugiyama, "Distribution of the largest latent root of the multivariate complex Gaussian distribution," Ann. Inst. Statist. Math., Vol. 24, pp. 87-94, 1972.

[34] I. E. Telatar, "Capacity of multi-antenna Gaussian channels," Eur. Trans. Telecom, Vol. 10, pp. 585-595, 1999.

[35] Q. T. Zhang and D. P. Liu, "A simple capacity formula for correlated diversity Rician fading channels," IEEE Communication Letters, Vol. 6, pp. 481-483, Nov. 2002. 